\title{
HOMOTOPY GROUPS OF THE OBSERVER MODULI SPACE OF RICCI POSITIVE METRICS
}

\author{
BORIS BOTVINNIK, MARK G. WALSH, AND DAVID J. WRAITH
}

\begin{abstract}
The observer moduli space of Riemannian metrics is the quotient of the space $\mathcal{R}(M)$ of all Riemannian metrics on a manifold $M$ by the group of diffeomorphisms $\operatorname{Diff}_{x_{0}}(M)$ which fix both a basepoint $x_{0}$ and the tangent space at $x_{0}$. The group $\operatorname{Diff}_{x_{0}}(M)$ acts freely on $\mathcal{R}(M)$ providing $M$ is connected. This offers certain advantages over the classic moduli space, which is the quotient by the full diffeomorphism group. Results due to Botvinnik, Hanke, Schick and Walsh, and to Hanke, Schick and Steimle have demonstrated that the higher homotopy groups of the observer moduli space $\mathcal{M}_{x_{0}}^{s>0}(M)$ of positive scalar curvature metrics are, in many cases, non-trivial. The aim in the current paper is to establish similar results for the moduli space $\mathcal{M}_{x_{0}}^{\mathrm{Ric}>0}(M)$ of metrics with positive Ricci curvature. In particular we show that for a given $k$, there are infinite order elements in the homotopy group $\pi_{4 k} \mathcal{M}_{x_{0}}^{\text {Ric }>0}\left(S^{n}\right)$ provided the dimension $n$ is odd and sufficiently large. In establishing this we make use of a gluing result of Perelman. We provide full details of the proof of this gluing theorem, which we believe have not appeared before in the literature. We also extend this to a family gluing theorem for Ricci positive manifolds.
\end{abstract}

\section{Contents}

1. Introduction 2

1.1. Motivation and main result 2

1.2. The observer moduli spaces of metrics 3

1.3. The universal fibre metric 4

1.4. The work of Farrell and Hsiang 5

2. Gluing manifolds and a theorem of Perelman 6

2.1. The gluing construction 6

2.2. The theorem of Perelman 7

2.3. A family version of Perelman's Theorem 13

3. Hatcher bundles 14

3.1. Goette's Theorem 14

3.2. Preliminary constructions 14

3.3. Recollection of the $J$-homomorphism 17

3.4. The Hatcher bundle $E_{\lambda} \quad 19$

4. The fibrewise Ricci positive metric construction 19

4.1. Foreword 19

4.2. The metric on $D^{m} \times D^{n} \quad 20$

4.3. Specifying the ellipsoid 21

4.4. Normal curvatures of the ellipsoid $\quad 22$

4.5. Proof of the Main Theorem 25

References

Date: December 19, 2017.

2000 Mathematics Subject Classification. 53C27, 57R65, 58J05, 58J50.

Key words and phrases. Positive Ricci metrics, Hatcher bundles, Moduli spaces. 


\section{INTRODUCTION}

1.1. Motivation and main result. In recent years, there have been great efforts made to better understand the topology of moduli spaces of Riemannian metrics of positive scalar curvature on a smooth compact (usually spin) manifold; see [6, 5, 4, 16]. Apart from results of Kreck and Stolz in [17] and Wraith in [23] concerning path-connectivity, we know very little about topology of the corresponding moduli spaces of positive Ricci curvature metrics. (In this context we should also mention work of Dessai, Klaus and Tuschmann on moduli spaces of non-negative sectional curvature metrics in [8], and the results of Crowley, Schick and Steimle on the space of Ricci positive metrics on certain manifolds, see [7].) Whether or not there is any non-triviality in the higher homotopy groups of such moduli spaces is still an open question. Here we study the topology of its closest relative, the observer moduli space $\mathcal{M}_{x_{0}}^{\text {Ric }>0}\left(S^{n}\right)$ of positive Ricci curvature metrics on the sphere $S^{n}$.

We denote by $d s_{n}^{2}$ the standard round metric on $S^{n}$, and by $\left[d s_{n}^{2}\right]$ its orbit in the moduli space $\mathcal{M}_{x_{0}}^{\mathrm{Ric}>0}\left(S^{n}\right)$. Here is our main result:

Main Theorem. For any $m \in \mathbb{N}$, there is an integer $N(m)$ such that for all odd $n>N(m)$, the group $\pi_{i}\left(\mathcal{M}_{x_{0}}^{\text {Ric }>0}\left(S^{n}\right),\left[d s_{n}^{2}\right]\right) \otimes \mathbb{Q}$ is non-trivial when $i=4 k$ and $k \leq m$.

We would like to emphasize that the observer moduli space is indeed the most tractable moduli space of metrics. Let $\mathcal{R}(M)$ be the space of all metrics on a compact closed manifold $M$, and $\operatorname{Diff}(M)$ be the group of diffeomorphisms which acts naturally on $\mathcal{R}(M)$ by pull-back. Even though the space $\mathcal{R}(M)$ is contractible, the moduli space of all metrics, i.e. the orbit space $\mathcal{R}(M) / \operatorname{Diff}(M)$, could be very complicated since some metrics have non-trivial isometry groups. Hence, in general, the action of $\operatorname{Diff}(M)$ on the space of metrics $\mathcal{R}(M)$ is far from being tractable. Following ideas from Gauge Theory, we fix an observer, i.e. a base point $x_{0} \in M$ together with a frame at the tangent space $T_{x_{0}} M$. Then we obtain the observer moduli space $\mathcal{M}_{x_{0}}(M):=\mathcal{R}(M) / \operatorname{Diff}_{x_{0}}(M)$, where the gauge group $\operatorname{Diff}_{x_{0}}(M)$ fixes such an observer. It is easy to see that the gauge group $\operatorname{Diff}_{x_{0}}(M)$ acts freely on the space of metrics provided $M$ is a connected manifold. Then the observer moduli space $\mathcal{M}_{x_{0}}(M)$ is homotopy equivalent to the classifying space $\mathrm{BDiff}_{x_{0}}(M)$, and the corresponding observer moduli space $\mathcal{M}_{x_{0}}^{\text {Ric }>0}(M)$ of positive Ricci metrics maps naturally to $\mathcal{M}_{x_{0}}(M)$, see below for more details.

The proof of Main Theorem is based on an analogous theorem by Botvinnik, Hanke, Schick and Walsh for the observer moduli space of positive scalar curvature metrics; see [4]. Both proofs rely heavily on work of Farrell, Hsiang, Hatcher and Goette; see [10] and [12]. Techniques for constructing families of metrics are also required. In the scalar curvature case, this means a family version of the Gromov-Lawson surgery technique from [11], described in [22]. Due to the flexibility of the scalar curvature and the strength of the Gromov-Lawson construction, this technique permits the detection of non-triviality for manifolds besides the sphere. Unsurprisingly, the Ricci curvature case requires a more delicate construction, which is based on a gluing theorem of Perelman. As yet, we have not demonstrated non-triviality beyond the case of the sphere. 
1.2. The observer moduli spaces of metrics. Let $M$ be a smooth closed connected manifold of dimension $n$. We denote by $\mathcal{R}(M)$, the space of all Riemannian metrics on $M$, equipped with the smooth Whitney topology. For a metric $g \in \mathcal{R}(M)$, we denote by $s_{g}$ and Ric $g$ its scalar and Ricci curvatures. We then consider the subspaces

$$
\mathcal{R}^{s>0}(M) \subset \mathcal{R}(M) \text { and } \quad \mathcal{R}^{\operatorname{Ric}>0}(M) \subset \mathcal{R}(M),
$$

of metrics with positive scalar and positive Ricci curvatures respectively. Let $\operatorname{Diff}(M)$ be the group of diffeomorphisms on $M$. This group acts on the space of metrics by pull-back:

$$
\operatorname{Diff}(M) \times \mathcal{R}(M) \rightarrow \mathcal{R}(M), \quad(\phi, g) \mapsto \phi^{*} g .
$$

Recalling that $M$ is connected, we fix a base point $x_{0} \in M$ which plays the role of an observer in a sense which will become clear shortly. Let $\operatorname{Diff}_{x_{0}}(M) \subset \operatorname{Diff}(M)$ be the subgroup of diffeomorphisms $\phi: M \rightarrow M$ such that $\phi\left(x_{0}\right)=x_{0}$ and such that the derivative $d \phi_{x_{0}}: T_{x_{0}} M \rightarrow T_{x_{0}} M$ is the identity. This is the observer diffeomorphism group of $M$ based at $x_{0}$.

As we have mentioned, the group $\operatorname{Diff}_{x_{0}}(M)$ acts freely on the space of metrics $\mathcal{R}(M)$ provided $M$ is a connected manifold; see [4, Lemma 1.2]. The orbit space $\mathcal{M}_{x_{0}}(M):=\mathcal{R}(M) /$ Diff $_{x_{0}}$ is the observer moduli space of metrics on $M$. Since the space $\mathcal{R}(M)$ is contractible and the action of $\operatorname{Diff}_{x_{0}}(M)$ on $\mathcal{R}(M)$ is proper, see [9, Lemma 1.2], the observer moduli space $\mathcal{M}_{x_{0}}(M)$ is homotopy equivalent to the classifying space $\operatorname{BDiff}_{x_{0}}(M)$ of the group $\operatorname{Diff}_{x_{0}}(M)$. In particular, we have a $\operatorname{Diff}_{x_{0}}(M)$-principal bundle:

$$
\operatorname{Diff}_{x_{0}}(M) \rightarrow \mathcal{R}(M) \rightarrow \mathcal{M}_{x_{0}}(M) .
$$

By restricting the action of $\operatorname{Diff}_{x_{0}}(M)$ to the appropriate subspaces, we obtain the observer moduli spaces

$$
\mathcal{M}_{x_{0}}^{s>0}(M):=\mathcal{R}^{s>0}(M) / \operatorname{Diff}_{x_{0}}(M), \quad \mathcal{M}_{x_{0}}^{\mathrm{Ric}>0}(M):=\mathcal{R}^{\mathrm{Ric}>0}(M) / \operatorname{Diff}_{x_{0}}(M),
$$

of positive scalar and of positive Ricci curvature metrics respectively. The inclusions of spaces of metrics $\mathcal{R}^{\mathrm{Ric}>0}(M) \subset \mathcal{R}^{s>0}(M) \subset \mathcal{R}(M)$ then induce the maps of principal $\operatorname{Diff}_{x_{0}}(M)$-bundles:

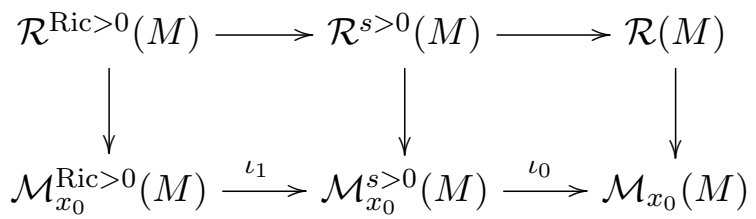

We denote $\iota:=\iota_{0} \circ \iota_{1}: \mathcal{M}_{x_{0}}^{\text {Ric }>0}(M) \rightarrow \mathcal{M}_{x_{0}}(M)$. The fibre bundles 1.1 give rise to the following commutative diagram, where the horizontal lines are Serre fibrations:

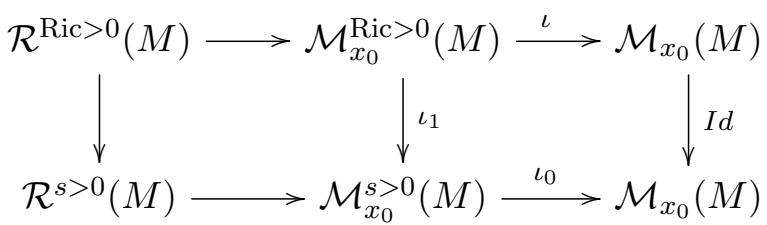


Letting $g_{0}$ denote a base point metric in $\mathcal{R}(M)$, we consider the induced diagram of homotopy group homomorphisms below:

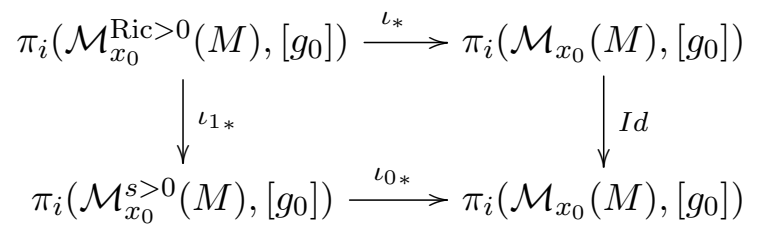

It is well-known that an element in the homotopy group $\pi_{i}\left(\mathcal{M}_{x_{0}}(M),\left[g_{0}\right]\right)$ can be represented by a smooth fibre bundle $E \rightarrow S^{i}$ with a fibre $M$. Hence to show that such an element lies in the image of $\iota_{0 *}$, it is enough to show that there exists a metric on the total space $E$ which restricts to a psc-metric on every fibre, see [4]. Here our task is more difficult: we have to construct such a metric on $E$ which is fibre-wise Ricci-positive, and the methods used involve geometric constructions which are quite different from the positive scalar curvature case. This is one of the reasons why we restrict our attention to the case when $M=S^{n}$. Next, we focus on the geometrical properties of the moduli space $\mathcal{M}_{x_{0}}(M)$.

1.3. The universal fibre metric. As we have mentioned earlier, the observer moduli space $\mathcal{M}_{x_{0}}(M)$ is homotopy equivalent to the classifying space $\operatorname{BDiff}_{x_{0}}(M)$.

We say that a fibre bundle $E \rightarrow X$ with fibre $M$ is a smooth $M$-fibre bundle if its structure group is a subgroup of $\operatorname{Diff}_{x_{0}}(M)$. Now we consider the universal principal bundle $\mathcal{R}(M) \rightarrow \mathcal{M}_{x_{0}}(M)$. Here the group $\operatorname{Diff}_{x_{0}}(M)$ acts freely on $\mathcal{R}(M)$, and the Borel construction gives the universal smooth $M$-fibre bundle $E(M) \rightarrow \mathcal{M}_{x_{0}}(M)$, where $E(M):=\mathcal{R}(M) \times_{\operatorname{Diff}_{x_{0}}(M)} M$. Recall that the space $\mathcal{R}(M) \times{ }_{\operatorname{Diff}_{x_{0}}(M)} M$ is defined as the quotient of $\mathcal{R}(M) \times M$ by the action of $\operatorname{Diff}_{x_{0}}(M)$ given by $\phi .(h, x)=\left(\left(\phi^{-1}\right)^{*} h, \phi(x)\right)$, where $\phi \in \operatorname{Diff}_{x_{0}}(M), h \in \mathcal{R}(M)$ and $x \in M$.

Given that $X$ is a paracompact Hausdorff space, recall that the isomorphism classes of principal $\operatorname{Diff}_{x_{0}}(M)$-bundles over $X$ are in one to one correspondence with homotopy classes $\left[X, \mathcal{M}_{x_{0}}(M)\right]$ of maps $X \rightarrow \mathcal{M}_{x_{0}}(M)$. In particular, given a map $f: X \rightarrow \mathcal{M}_{x_{0}}(M)$, we obtain a commutative diagram:

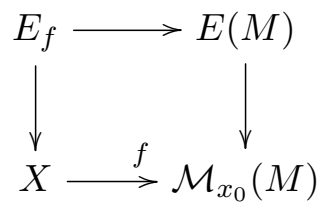

where the bundle $E_{f} \rightarrow X$ is the pull-back of the universal smooth $M$-fibre bundle by the map $f$.

There is however a more refined structure which we can associate to such a bundle. The total space $E(M)=\mathcal{R}(M) \times{ }_{\text {Diff }_{x_{0}}(M)} M$ admits a "universal fibre metric" which we will now define. We begin with an arbitrary point $[h, x] \in \mathcal{R}(M) \times_{\operatorname{Diff}_{x_{0}}(M)} M$. The fibre at this point is of course diffeomorphic to $M$. Let us now consider the tangent space to this fibre. Suppose $(h, x),\left(h^{\prime}, x^{\prime}\right) \in$ $\mathcal{R}(M) \times M$ both represent the point $[h, x] \in \mathcal{R}(M) \times \operatorname{Diff}_{x_{0}(M)} M$. Then the tangent spaces $T_{x} M$ and $T_{x^{\prime}} M$ are isomorphically related by the derivative map $\phi_{*}$ of some diffeomorphism $\phi \in \operatorname{Diff}_{x_{0}} M$ which satisfies $\phi(x)=x^{\prime}$. Thus, the tangent space to $[h, x]$ can be thought of as the isomorphic 
identification of all tangent spaces $T_{x^{\prime}} M$ where $x^{\prime} \in M$ lies in the orbit of $x$ under the action of $\operatorname{Diff}_{x_{0}} M$. Suppose now that $[u],[v]$ denote tangent vectors to the fibre at $[x, h]$ represented by tangent vectors $u, v \in T_{x} M$. We specify an inner product to the tangent space to the fibre at $[h, x]$ by the following formula:

$$
\langle[u],[v]\rangle_{[x, h]}=h_{x}(u, v)
$$

where $h_{x}$ is the restriction of the Riemannian metric $h$ to the tangent space $T_{x} M$. It is an easy exercise to show that this is well-defined and varies smoothly over $E(M)$; see [21, p. 61]. Notice that this does not give a Riemannian metric on $E(M)$ as we only specify the inner product on fibres.

Given a map $f: X \rightarrow \mathcal{M}_{x_{0}}(M)$, this universal fibre metric then pulls back to a continuous fibrewise family of Riemannian metrics on $E_{f}$. More precisely, each fibre of the bundle $E_{f} \rightarrow X$, already diffeomorphic to $M$, is now equipped with a Riemannian metric which depends continuously on $X$. Clearly, varying the map $f$ by a homotopy alters the fibrewise metric structure of the bundle. Suppose, on the other hand, we begin with a fibrewise family of metrics on an $M$-bundle over $X$. Identifying fibres non-canonically with a 'standard' copy of $M$ and pulling back metrics leads to a well-defined map $X \rightarrow \mathcal{M}_{x_{0}}(M)$. Thus, we obtain a one to one correspondence between maps $X \rightarrow \mathcal{M}_{x_{0}}(M)$ and fibrewise families of metrics on $M$ which are parameterised by $X$.

Assuming $X$ is the sphere $S^{i}$, we are now brought back to the homomorphism of homotopy groups

$$
\iota_{*}: \pi_{i}\left(\mathcal{M}_{x_{0}}^{\mathrm{Ric}>0}(M),\left[g_{0}\right]\right) \longrightarrow \pi_{i}\left(\mathcal{M}_{x_{0}}(M),\left[g_{0}\right]\right),
$$

induced by the inclusion $\iota: \mathcal{M}_{x_{0}}^{\text {Ric }>0}(M) \hookrightarrow \mathcal{M}_{x_{0}}(M)$. Let $f: S^{i} \rightarrow \mathcal{M}_{x_{0}}(M)$ represent an element of $\pi_{i}\left(\mathcal{M}_{x_{0}}(M),\left[g_{0}\right]\right)$. This element determines (and is determined by) an $M$-bundle $E_{f} \rightarrow S^{i}$ as above, together with a fibrewise family of metrics on $E_{f}$. Thus, it is possible to lift this element of $\pi_{i}\left(\mathcal{M}_{x_{0}}(M),\left[g_{0}\right]\right)$ to an element of $\pi_{i}\left(\mathcal{M}_{x_{0}}^{\text {Ric }>0}(M),\left[g_{0}\right]\right)$, provided we can construct a fibrewise family of positive Ricci curvature metrics on $E_{f}$.

1.4. The work of Farrell and Hsiang. At this stage we have established that lifting an element of $\pi_{i}\left(\mathcal{M}_{x_{0}}(M),\left[g_{0}\right]\right)$ to $\pi_{i}\left(\mathcal{M}_{x_{0}}^{\text {Ric }>0}(M),\left[g_{0}\right]\right)$ involves the construction of a family of fiberwise Ricci positive Riemannian metrics on some bundle over $S^{i}$. However, we have not yet discussed the particular elements in the homotopy groups of $\mathcal{M}_{x_{0}}(M)$ which we plan to lift. It is here that we recall a result of Farrell and Hsiang, which identifies the rational homotopy groups of BDiff $x_{x_{0}}\left(S^{n}\right)$ in a stable range, using algebraic $K$-theory and Waldhausen $K$-theory computations; see [10]. Recalling that $\mathcal{M}_{x_{0}}\left(S^{n}\right)$ is homotopy equivalent to the classifying space BDiff $x_{0}\left(S^{n}\right)$, the result of these computations can be stated as follows.

Theorem 1. (Farrell and Hsiang, [10.) For any $m \in \mathbb{N}$, there is an integer $N(m)$ such that for all odd $n>N(m)$ and $i \leq 4 m$,

$$
\pi_{i} \mathcal{M}_{x_{0}}\left(S^{n}\right) \otimes \mathbb{Q}= \begin{cases}\mathbb{Q} & \text { if } n \text { odd and } i \equiv 0 \bmod 4 \\ 0 & \text { otherwise }\end{cases}
$$


Thus, for appropriate $i$, we now have lots of non-trivial groups $\pi_{i}\left(\mathcal{M}_{x_{0}}\left(S^{n}\right),\left[g_{0}\right]\right) \otimes \mathbb{Q}$. This also explains the hypotheses of the main theorem.

This leaves the question of which $S^{n}$-bundles over $S^{i}(i \equiv 0 \bmod 4)$ can represent the nontrivial elements of $\pi_{i}\left(\mathcal{M}_{x_{0}}\left(S^{n}\right),\left[g_{0}\right]\right) \otimes \mathbb{Q}$. It turns out that these elements can be represented by so-called 'Hatcher bundles'. In section 3 we will provide a description of these, based on the work of Hatcher and Goette (see [12]). Our approach to the topological construction of Hatcher bundles is guided by the geometric constructions we must subsequently perform, namely the production of fibrewise Ricci positive metrics. These metric issues will be addressed in section 4 , and will involve a generalized version of a powerful gluing theorem due to Perelman. Perelman's theorem and our generalization of this is the subject of section 2 .

This work was initiated while the third named author was visiting the first, and he would like to thank the University of Oregon for their hospitality. He would also like to thank Sebastian Goette for a useful discussion about Hatcher bundles, and Janice Love for her help with the Maple code used in section 2 ,

\section{Gluing manifolds And a theorem of Perelman}

2.1. The gluing construction. The purpose of this section is to present a theorem of Perelman which allows for the construction of Ricci positive metrics on a closed manifold by gluing together certain Ricci positive metrics on manifolds with boundary. This result is the principal geometric tool used in achieving our goal of obtaining a fibrewise family of positive Ricci curvature metrics on the total space of a Hatcher bundle. Perelman's theorem is originally published in [19] and justified with a brief outline, omitting the details. Our aim is to provide those details, and in so doing offer a more comprehensive justification, currently lacking in the literature, for a very useful result. In our experience this result is not widely known, and we hope that by offering these details we will help provide some of the intuition behind the construction, as well as bringing it to a potentially wider audience. Moreover, these details are important for establishing the family version of Perelman's theorem, which appears at the end of this section.

We begin with a brief review of the notion of gluing smooth manifolds, something we make extensive use of throughout the paper. Consider a pair of smooth $n$-dimensional manifolds, $M_{1}$ and $M_{2}$, each with non-empty boundary. We further assume that $\partial M_{1}$ and $\partial M_{2}$ are diffeomorphic via a diffeomorphism $\phi: \partial M_{1} \rightarrow \partial M_{2}$. From this, we may form the adjunction space, $W=M_{1} \cup_{\phi} M_{2}$, obtained as the quotient of $M_{1} \sqcup M_{2}$ by identifying each $x \in \partial M_{1}$ with $\phi(x) \in \partial M_{2}$. In particular, the quotient map embeds both $M_{1}$ and $M_{2}$ into this space. For simplicity then, we identify $M_{1}$ and $M_{2}$ with their images in $W$ and write $X=\partial M_{1}=\partial M_{2}$. Consider collar neighbourhoods $\partial M_{1} \times(-\delta, 0]$ and $\partial M_{2} \times[0, \delta)$ about $X$ for some small $\delta>0$, for example determined by the normal coordinate from $\partial M_{1}, \partial M_{2}$ with respect to some choice of metrics on $M_{1}, M_{2}$. Denote by $N$ the union of the images of these collar neighbourhoods in $W$. We then have a homeomorphism 
between $X \times(-\delta, \delta)$ and $N$ given by

$$
(x, r) \mapsto\left\{\begin{array}{cc}
\left(m_{1}, r\right) & \text { if } r \leq 0 \\
\left(\phi\left(m_{1}\right), r\right) & \text { if } r \geq 0,
\end{array}\right.
$$

where $x \in X$ is the equivalence class $x=\left\{m_{1}, \phi\left(m_{1}\right)\right\}$ for some $m_{1} \in \partial M_{1}$.

We can now use this to give $N$ a differentiable structure, by pulling back the standard differentable structure on $X \times(-\delta, \delta)$ via the inverse homeomorphism. Finally, we extend this differentiable structure over $M_{1}$ and $M_{2}$ to give a differentiable structure on $W$. Although there are many choices involved in this construction, leading to many possible differentiable structures, it is a well known fact that the diffeomorphism type of the resulting smooth manifold $W$ is independent of these choices; see [15, Ch. 8, Sec. 2]. We now consider such a gluing in the Riemannian setting,
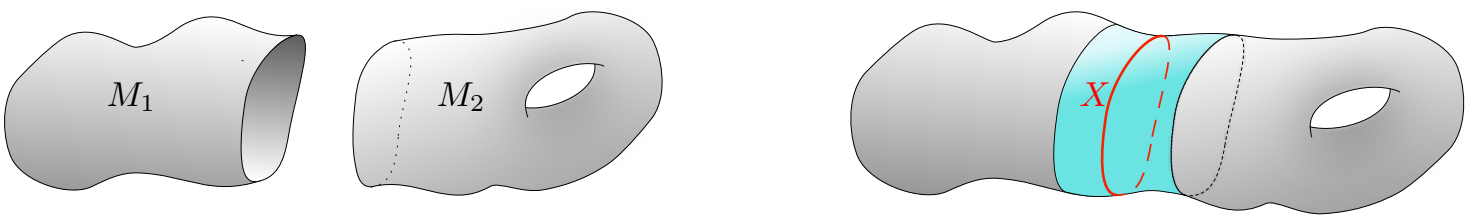

FiguRE 1. The manifolds with boundary, $M_{1}$ and $M_{2}$ (left) along with the adjunction space $W$ and tubular neighborhood $N$ of $X \subset W$ (right)

equipping $M_{1}$ and $M_{2}$ with Riemannian metrics, $h_{1}$ and $h_{2}$. Let us assume that the restrictions of these metrics to their respective boundaries are isometric via $\phi$. More precisely, we assume

$$
\left.h_{1}\right|_{\partial M_{1}}=\left.\phi^{*} h_{2}\right|_{\partial M_{2}} .
$$

This automatically leads to a well-defined $C^{0}$-metric $h=h_{1} \cup_{\phi} h_{2}$, on $M_{1} \cup_{\phi} M_{2}$. Notice that this adjunction metric is smooth if and only if it is smooth in a collar neighbourhood of $X \subset M_{1} \cup_{\phi} M_{2}$. In view of the of the adjunction space discussion above, this will be the case if the metric $\left.h_{1}\right|_{\partial M_{1} \times(-\delta, 0]}$ glues smoothly with $\left(\phi \times i d_{[0, \delta)}\right)^{*}\left(\left.h_{2}\right|_{\partial M_{2} \times[0, \delta)}\right)$.

2.2. The theorem of Perelman. The above construction gives the $C^{0}$-metric $h_{1} \cup_{\phi} h_{2}$ on $M_{1} \cup M_{2}$. We will be interested in smoothing the metric $h_{1} \cup_{\phi} h_{2}$ within positive Ricci curvature in the case where $h_{1}$ and $h_{2}$ individually have positive Ricci curvature. This is not always possible. However, the following theorem of Perelman shows that under certain additional assumptions involving the normal (i.e. the principal) curvatures of $h_{1}$ and $h_{2}$ at the boundary, such a smoothing can be performed.

Theorem 2. Let $\left(M_{1}, h_{1}\right)$ and $\left(M_{2}, h_{2}\right)$ be a pair of Riemannian manifolds with positive Ricci curvature and $\phi:\left(\partial M_{1},\left.h_{1}\right|_{\partial M_{1}}\right) \rightarrow\left(\partial M_{2},\left.h_{2}\right|_{\partial M_{2}}\right)$, an isometry of their boundaries. Suppose that the normal curvatures of $\left.h_{1}\right|_{\partial M_{1}}$ with respect to the outward normal are greater than the negatives of corresponding normal curvatures of $\left.h_{2}\right|_{\partial M_{2}}$ with respect to its outward normal. Then the $C^{0}$-metric, $h=h_{1} \cup_{\phi} h_{2}$ on the smooth manifold $M_{1} \cup_{\phi} M_{2}$ can be replaced by a $C^{\infty}$-metric with positive Ricci curvature, agreeing with $h_{1}$ and $h_{2}$ outside a neighbourhood of the glued boundaries. 
Proof. As above, we will denote by $X$ the hypersurface of $M_{1} \cup_{\phi} M_{2}$ along which $M_{1}$ and $M_{2}$ are joined, and assume that the normal parameter through the hypersurface $X$ gives rise to collar neighbourhoods $\partial M_{2} \times[0, \delta)$ in $M_{2}$ and $\partial M_{1} \times(-\delta, 0]$ in $M_{1}$, for some $\delta>0$. Since we will be working exclusively in a collar neighbourhood of $X$, for convenience we can simply re-label the metric $\left(\phi \times i d_{[0, \delta)}\right)^{*}\left(\left.h_{2}\right|_{\partial M_{2} \times[0, \delta)}\right)$ by $h_{2}$, assume that $\partial M_{1}=\partial M_{2}$ and that $\phi$ is the identity map. Thus from now on we will write $h_{1} \cup h_{2}$ for the $C^{0}$-metric in the theorem, and $M_{1} \cup M_{2}$ for the manifold. We will introduce a parameter $t$, normal to $X$, running from $M_{1}$ to $M_{2}$, such that $t=0$ corresponds to $X$. Observe that $M_{1} \cup M_{2}$ has a smooth topological structure (though not a smooth metric structure), and that with respect to this $t$ is a smooth parameter.

Choose a small parameter $\epsilon>0$. (We will say more about an appropriate size for $\epsilon$ later.) Our next task is to write down a new metric on $X \times[-\epsilon, \epsilon]$ which joins with $h_{1}$ for $t<\epsilon$ and $h_{2}$ for $t>\epsilon$ to give a $C^{1}$-metric on $M_{1} \cup M_{2}$. This new metric will take the form $d t^{2}+g(t)$. Denoting by $h_{i}(t)$, where $i=1$ or 2 , the induced metric on the hypersurface of constant distance $t$ from $X$, we will choose $g(t)$ to be the following cubic expression in $t$ :

$$
\begin{aligned}
g(t)= & \frac{t+\epsilon}{2 \epsilon} h_{2}(\epsilon)-\frac{t-\epsilon}{2 \epsilon} h_{1}(-\epsilon)+\frac{(t-\epsilon)^{2}(t+\epsilon)}{4 \epsilon^{2}}\left[h_{1}^{\prime}(-\epsilon)-\frac{1}{2 \epsilon}\left[h_{2}(\epsilon)-h_{1}(-\epsilon)\right]\right] \\
& +\frac{(t+\epsilon)^{2}(t-\epsilon)}{4 \epsilon^{2}}\left[h_{2}^{\prime}(\epsilon)-\frac{1}{2 \epsilon}\left[h_{2}(\epsilon)-h_{1}(-\epsilon)\right]\right] .
\end{aligned}
$$

Lemma 1. Assume that the metrics $h_{1}, h_{2}$ satisfy the hypothesis from Theorem 2 . Then there exists $\epsilon>0$ such that

(i) with $g(t)$ as in 2.1), the metric $d t^{2}+g(t)$ is smooth if $t \neq 0$ and $C^{1}$ at $t=0$;

(ii) $\operatorname{Ric}_{d t^{2}+g(t)}>0$.

Proof of Lemma 1. First, we find the $t$-derivative of this metric. A straightforward calculation gives

$$
\begin{aligned}
g^{\prime}(t)= & \frac{1}{2 \epsilon}\left[h_{2}(\epsilon)-h_{1}(-\epsilon)\right]+\frac{2\left(t^{2}-\epsilon^{2}\right)+(t-\epsilon)^{2}}{4 \epsilon^{2}}\left[h_{1}^{\prime}(-\epsilon)-\frac{1}{2 \epsilon}\left[h_{2}(\epsilon)-h_{1}(-\epsilon)\right]\right] \\
& +\frac{2\left(t^{2}-\epsilon^{2}\right)+(t+\epsilon)^{2}}{4 \epsilon^{2}}\left[h_{2}^{\prime}(\epsilon)-\frac{1}{2 \epsilon}\left[h_{2}(\epsilon)-h_{1}(-\epsilon)\right]\right] .
\end{aligned}
$$

It is now an easy exercise to check that the metric $g$ forms a $C^{1}$ join with the $h_{i}$ at $t= \pm \epsilon$. (The metric $g$ is of course smooth.)

Our next task is to investigate the curvature properties of $d t^{2}+g(t)$. We begin by assuming that the cubic expression for $g(t)$ above holds in an open neighbourhood containing $[-\epsilon, \epsilon] \times X$. Motivated by the fact that curvature is a second derivative phenomenon, an easy calculation shows that

$$
g^{\prime \prime}(t)=\frac{1}{4 \epsilon^{2}}(6 t-2 \epsilon)\left[h_{1}^{\prime}(-\epsilon)-\frac{1}{2 \epsilon}\left[h_{2}(\epsilon)-h_{1}(-\epsilon)\right]\right]+\frac{1}{4 \epsilon^{2}}(6 t+2 \epsilon)\left[h_{2}^{\prime}(\epsilon)-\frac{1}{2 \epsilon}\left[h_{2}(\epsilon)-h_{1}(-\epsilon)\right]\right] .
$$


We will investigate the limiting behaviour of $g^{\prime \prime}( \pm \epsilon)$ as $\epsilon \rightarrow 0$. At $t=\epsilon$ we have

$$
g^{\prime \prime}(\epsilon)=\frac{1}{\epsilon}\left[h_{1}^{\prime}(-\epsilon)+2 h_{2}^{\prime}(\epsilon)-\frac{3}{2} \frac{h_{2}(\epsilon)-h_{1}(-\epsilon)}{\epsilon}\right] .
$$

Consider the term $\left(h_{2}(\epsilon)-h_{1}(-\epsilon)\right) / \epsilon$ in $(2.2)$. In the limit $\epsilon \rightarrow 0$ we see by l'Hôpital's rule that the value of the limit is $h_{1}^{\prime}(0)+h_{2}^{\prime}(0)$. Clearly, the overall limit of the bracketed term in 2.2 is

$$
h_{1}^{\prime}(0)+2 h_{2}^{\prime}(0)-\frac{3}{2}\left(h_{1}^{\prime}(0)+h_{2}^{\prime}(0)\right)=\frac{1}{2}\left(h_{2}^{\prime}(0)-h_{1}^{\prime}(0)\right) .
$$

A similar calculation shows that the corresponding term in $\lim _{\epsilon \rightarrow 0} g^{\prime \prime}(-\epsilon)$ yields exactly the same expression.

Next observe that $g^{\prime \prime}(t)$ has a linear dependence on $t$. Thus if $g^{\prime \prime}(-\epsilon)$ and $g^{\prime \prime}(\epsilon)$ have the same sign, then this sign persists for all $t \in[-\epsilon, \epsilon]$. We will show that under the Perelman normal curvature assumption, the sign of both is negative provided $\epsilon$ is chosen sufficiently small 1

Assume that $\epsilon$ is chosen so small that the topological product structure in a neighbourhood of $X$ extends over $t \in[-2 \epsilon, 2 \epsilon]$. Let $u$ be a fixed vector tangent to $X$ at some point $x_{0}$. As $u$ is independent of the metric, given the product structure around $X$, we can consider the 'same' vector for nearby values of $t$, i.e. we obtain a local vector field $u$ along the line $\left(t, x_{0}\right)$. Define the normal curvature function $k(u)$ to be $k(u)=\left\langle\nabla_{u} \partial_{t}, u\right\rangle$, which is the normal curvature for the vector $u$ of the hypersurface $t=$ constant, with $\partial / \partial t$ providing the normal direction. Note that we do not insist that $u$ is unit with respect to any metric. But now observe that we can rearrange this definition to give $k(u)=\frac{1}{2} \frac{\partial}{\partial t}\langle u, u\rangle$, which is just $\frac{1}{2} g^{\prime}(t)(u, u)$. Differentiating with respect to $t$ we obtain $k^{\prime}(u)=\frac{1}{2} g^{\prime \prime}(t)(u, u)$.

The difference of the normal curvatures corresponding to $u$ across $X$ can be viewed as

$$
\frac{1}{2} \lim _{\epsilon \rightarrow 0}\left(g^{\prime}(\epsilon)(u, u)-g^{\prime}(-\epsilon)(u, u)\right)=\frac{1}{2}\left(h_{1}^{\prime}(0)-h_{2}^{\prime}(0)\right) .
$$

Denoting the normal curvatures at $\partial M_{1}$ and $\partial M_{2}$ with respect to the outward normals by $k_{1}, k_{2}$, it is straightforward to see that

$$
\begin{array}{ll}
k_{1}(u)=\lim _{t \rightarrow 0^{-}} k(u) & =\frac{1}{2} \lim _{t \rightarrow 0^{-}} g^{\prime}(t)(u, u) ; \\
k_{2}(u)=-\lim _{t \rightarrow 0^{+}} k(u) & =-\frac{1}{2} \lim _{t \rightarrow 0^{+}} g^{\prime}(t)(u, u) .
\end{array}
$$

Thus the difference of normal curvatures 2.3 is equal to $k_{1}(u)+k_{2}(u)$. Now the Perelman normal curvature assumption is that $k_{1}(u)>-k_{2}(u)$, which means that the difference of normal curvatures is positive, and hence $\lim _{\epsilon \rightarrow 0} \epsilon g^{\prime \prime}( \pm \epsilon)=\frac{1}{2}\left(h_{2}^{\prime}(0)-h_{1}^{\prime}(0)\right)<0$. Therefore by choosing $\epsilon$ sufficiently small, we can bound $g^{\prime \prime}(t)(u, u)$ as

$$
g^{\prime \prime}(t)(u, u)<-A \cdot|u|,
$$

where $-A$ is an arbitrarily large negative constant and the norm of $u$ is taken with respect to $h_{1}$ or $h_{2}$ say, as these are common on $X$. In turn this means that $k^{\prime}(u)$ can similarly be bounded above.

1 Notice what we have used so far. For the $C^{1}$ cubic expression we require no assumptions. In order to obtain the limiting formula for the second derivative we only need that the original metric on the union is continuous at $t=0$. 
The relevance of $k^{\prime}(u)$ is that it can be re-written in terms of the curvature tensor applied to $u$ and $\partial_{t}:=\frac{\partial}{\partial t}$, and we can use the arbitrarily negative feature of $k^{\prime}(u)$ to produce an arbitrarily large positive lower bound for $R\left(\partial_{t}, u, u, \partial_{t}\right)$. In detail we have

$$
\begin{aligned}
k^{\prime}(u) & =\partial_{t}\left\langle\nabla_{u} \partial_{t}, u\right\rangle=\left\langle\nabla_{\partial_{t}} \nabla_{u} \partial_{t}, u\right\rangle+\left\langle\nabla_{u} \partial_{t}, \nabla_{\partial_{t}} u\right\rangle \\
& =\left\langle\nabla_{t} \nabla_{u} \partial_{t}, u\right\rangle+|S(u)|^{2}
\end{aligned}
$$

where $S(u)$ denotes the shape operator of the hypersurfaces given by constant values of $t$, and where we have used the fact that $\nabla_{\partial t} u=\nabla_{u} \partial t$ since $[\partial t, u] \equiv 0$. On the other hand we have

$$
\begin{aligned}
R\left(\partial_{t}, u, u, \partial_{t}\right) & =-R\left(\partial_{t}, u, \partial_{t}, u\right) \\
& =-\left[\left\langle\nabla_{\partial_{t}} \nabla_{u} \partial_{t}, u\right\rangle-\left\langle\nabla_{u} \nabla_{\partial_{t}} \partial_{t}, u\right\rangle\right] \\
& =-\left\langle\nabla_{\partial_{t}} \nabla_{u} \partial_{t}, u\right\rangle
\end{aligned}
$$

as $\nabla_{\partial_{t}} \partial_{t} \equiv 0$. Thus we conclude that

$$
R\left(\partial_{t}, u, u, \partial_{t}\right)=-k^{\prime}(u)+|S(u)|^{2} .
$$

In particular, since $k^{\prime}(u)=\frac{1}{2} g^{\prime \prime}(u, u)$, and $g^{\prime \prime}(t)(u, u)<-A|u|$, as in 2.4), for small enough $\epsilon>0$, we can bound $R\left(\partial_{t}, u, u, \partial_{t}\right)$ below by any given positive constant.

The observation is now that any Ricci curvature expression must contain this large positive term, and we therefore get positive Ricci curvature for the metric $d t^{2}+g(t)$, provided we show that other curvature tensor expressions remain bounded. It is easily checked that this boundedness reduces to showing that $\left.\| R\left(u_{i}, u_{j}\right) u_{k}\right) \|$ is bounded above by some constant independent of $\epsilon$ for all vectors $u_{i}, u_{j}, u_{k}$ tangent to $X$ which are unit with respect to say $h_{1}(0)=h_{2}(0)$.

With the above curvature expression 2.5 in mind, consider the derivatives of the metric $g(t)$ in directions orthogonal to $t$. The quantities $h_{1}(-\epsilon), h_{1}^{\prime}(-\epsilon), h_{2}(\epsilon), h_{2}^{\prime}(\epsilon)$ and their derivatives can clearly be bounded independent of $\epsilon$. We also know that the other terms involving $\epsilon$ :

$$
\frac{t \pm \epsilon}{2 \epsilon}, \quad \frac{1}{2 \epsilon}\left(h_{2}(\epsilon)-h_{1}(-\epsilon)\right), \quad \frac{(t \pm \epsilon)^{2}(t \mp \epsilon)}{4 \epsilon^{2}}
$$

all remain bounded for $t \in[-\epsilon, \epsilon]$ as $\epsilon \rightarrow 0$. Therefore the derivatives of $g(t)$ orthogonal to $t$ must stay bounded independent of $\epsilon$.

We also claim that the first derivative of $g(t)$ with respect to $t$ is bounded independently of $\epsilon$. This follows from the above calculations involving $g^{\prime \prime}( \pm \epsilon)$, see 2.2 . We showed that for $\epsilon$ sufficiently small, the sign of $g^{\prime \prime}(t)(u, u)$ is negative, from which we see that the values of $g^{\prime}(t)(u, u)$ must lie between those at $t= \pm \epsilon$, and hence are bounded independent of $\epsilon$. We also notice that boundedness can then also be deduced for $g^{\prime}(t)(u, v)$ via the polarization formula for inner products.

We conclude that the norm $\left\|R\left(u_{i}, u_{j}\right) u_{k}\right\|$ is bounded for all $t \in[-\epsilon, \epsilon]$ independent of $\epsilon$ provided the curvature $R\left(u_{i}, u_{j}\right) u_{k}$ does not depend on the second derivative of the metric with respect to $t$. Without loss of generality assume that $u_{i}, u_{j}, u_{k}$ are coordinate vector fields for some 
coordinate system on $X$ extended to a coordinate system in a neighbourhood of $X$ by the parameter $t$. The relevant expression for the components of $R\left(u_{i}, u_{j}\right) u_{k}$ in terms of Christoffel symbols is

$$
R_{i j k}^{l}=\partial_{i} \Gamma_{j k}^{l}-\partial_{j} \Gamma_{i k}^{l}+\sum_{m=1}^{n}\left(\Gamma_{j k}^{m} \Gamma_{i m}^{l}-\Gamma_{i k}^{m} \Gamma_{j m}^{l}\right),
$$

where $l$ runs over all possible subscripts including $t$. Since the Christoffel symbols in (2.6) have at most one derivative with respect to $t$, we obtain the desired boundedness property of $\left.\| R\left(u_{i}, u_{j}\right) u_{k}\right) \|$. Thus we fix small $\epsilon>0$ so that the metric $g(t)$ has positive Ricci curvature and is smooth if $t \neq 0$, and $C^{1}$ if $t=0$. This proves Lemma 1 .

Proof of Theorem 2 continued. Our next goal is to show how to effect a $C^{2}$-smoothing of $g(t)$ in some $\tau$-neighbourhoods of $t= \pm \epsilon, \tau<<\epsilon$. As we will make a very general construction, it will be convenient to locally re-parameterize, and work in an interval $[-\tau, \tau]$.

We start with an arbitrary $C^{1}$-function $f(t)$, which is smooth away from $t=0$, and is defined in some open set of the real line containing $[-\tau, \tau]$. Given such function $f(t)$, we will replace $f(t)$ for $t \in[-\tau, \tau]$ by a quintic polynomial $p(t)$ which will agree to second order with $f(t)$ at $t= \pm \tau$. By applying this idea to Riemannian metrics, we can create the desired $C^{2}$-metric by quintic interpolation, in exactly the same way that we created a $C^{1}$-metric using a cubic interpolation.

Let $p(t)=\sum_{n=0}^{5} c_{n} t^{n}$, and suppose that $f(\tau)=a_{0}, f(-\tau)=b_{0}, f^{\prime}(\tau)=a_{1}, f^{\prime}(-\tau)=b_{1}$, $f^{\prime \prime}(\tau)=a_{2}, f^{\prime \prime}(-\tau)=b_{2}$. Assuming that $p^{(i)}( \pm \tau)=f^{(i)}( \pm \tau)$ for $i=0,1,2$ yields a $(5 \times 5)$-linear system with the $c_{n}$ as the unknowns and the $a_{i}, b_{i}$ as coefficients. Solving this system (using Maple) shows that the polynomial $p(t)$ is uniquely determined by the above requirements, and is equal to

$$
\begin{aligned}
p(t)= & \frac{\tau^{2}\left(a_{2}-b_{2}\right)-3 \tau\left(a_{1}+b_{1}\right)+3\left(a_{0}-b_{0}\right)}{16 \tau^{5}} t^{5}-\frac{-\tau\left(a_{2}+b_{2}\right)+\left(a_{1}-b_{1}\right)}{16 \tau^{3}} t^{4} \\
& -\frac{\tau^{2}\left(a_{2}-b_{2}\right)-5 \tau\left(a_{1}+b_{1}\right)+5\left(a_{0}-b_{0}\right)}{8 \tau^{3}} t^{3}+\frac{-\tau\left(a_{2}+b_{2}\right)+3\left(a_{1}-b_{1}\right)}{8 \tau} t^{2} \\
& +\frac{\tau^{2}\left(a_{2}-b_{2}\right)-7 \tau\left(a_{1}+b_{1}\right)+15\left(a_{0}-b_{0}\right)}{16 \tau} t+\frac{\tau^{2}\left(a_{2}+b_{2}\right)-5 \tau\left(a_{1}-b_{1}\right)}{16}+\frac{a_{0}+b_{0}}{2} .
\end{aligned}
$$

With an eye towards curvature considerations when this quintic interpolation has been applied to Riemannian metrics, consider next the effect on $p(t)$ (for $t \in[-\tau, \tau]$ ) of letting $\tau \rightarrow 0$. As this limit is approached, the term involving $t^{5}$ in the above expression for $p(t)$ approaches $\frac{3}{16}\left(a_{0}-b_{0}\right) \frac{t^{5}}{\tau^{5}}$, the $t^{3}$ term approaches $-\frac{5}{8}\left(a_{0}-b_{0}\right) \frac{t^{3}}{\tau^{3}}$, and the first order term in $t$ contributes $\frac{15}{16}\left(a_{0}-b_{0}\right) \frac{t}{\tau}$.

Recall that $|t| \leq|\tau|$, hence the limits of the $t^{5}, t^{3}$, and $t$-terms are bounded by

$$
\frac{3}{16}\left|a_{0}-b_{0}\right|, \quad \frac{5}{8}\left|a_{0}-b_{0}\right|, \quad \text { and } \quad \frac{15}{16}\left|a_{0}-b_{0}\right|
$$

respectively. Since $|t| \leq|\tau|$, the degree four and two terms in $t$ contribute nothing in the limit, and the zeroth order term yields $\frac{a_{0}+b_{0}}{2}$. In our case we can say more, however. Clearly, the coefficients $a_{i}$ and $b_{i}$ are functions of $\tau$, i.e., $a_{i}=a_{i}(\tau)$ and $b_{i}=b_{i}(\tau)$ for $i=0,1,2$, and since $f(t)$ is assumed $C^{1}$ at $t=0$ we see that $\lim _{\tau \rightarrow 0} a_{j}(\tau)=\lim _{\tau \rightarrow 0} b_{j}(\tau)=f^{(j)}(0)$ for $j=0,1$. Thus we conclude that for $\tau$ sufficiently small, the polynomial $p(t)$ can $C^{0}$-approximate the constant function with value $\left(a_{0}+b_{0}\right) / 2=a_{0}=b_{0}=f(0)$ over the interval $[-\tau, \tau]$ to within any desired degree. 
Applying the same analysis to $p^{\prime}(t)$ shows that for $\tau$ sufficiently small, $p^{\prime}(t) C^{0}$-approximates the constant function with value $\left(a_{1}+b_{1}\right) / 2=a_{1}=b_{1}=f^{\prime}(0)$ over the interval $[-\tau, \tau]$ to within any desired degree. In other words, by choosing $\tau$ sufficiently small, $p(t)$ will $C^{1}$-approximate $f(t)$ over $[-\tau, \tau]$ to within any desired accuracy.

Finally, we must consider the behaviour of $p^{\prime \prime}(t)$. Analogous arguments to the above show that for $\tau$ sufficiently small, $p^{\prime \prime}(t)$ can be $C^{0}$-approximated over $[-\tau, \tau]$ to within any desired degree of accuracy by the cubic

$$
\frac{5}{4}\left(a_{2}-b_{2}\right) \frac{t^{3}}{\tau^{3}}-\frac{3}{4}\left(a_{2}-b_{2}\right) \frac{t}{\tau}+\frac{1}{2}\left(a_{2}+b_{2}\right) .
$$

To understand the behaviour of this cubic, it clearly suffices to examine the function $q(t)=\frac{5}{\tau^{3}} t^{3}-\frac{3}{\tau} t$ over $t \in[-\tau, \tau]$. An elementary calculation shows that maximum and minimum values taken by $q(t)$ over $[-\tau, \tau]$ are $q(\tau)=2$ respectively $q(-\tau)=-2$. This function is depicted in Fig. 2 below.

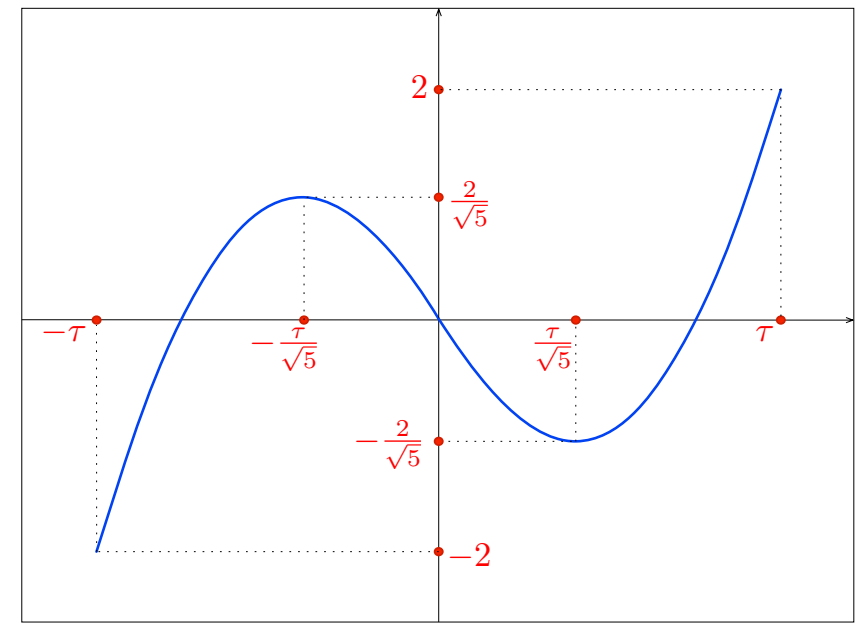

Figure 2. The graph of the function $q(t)$

Thus the values of $q(t)$ for all other $t$ in this interval lie between the endpoint values. It follows immediately that the same is true for $p^{\prime \prime}(t)$ (with respect to its endpoint values).

Lemma 2. Let $g(t)$ be the $C^{1}$-metric given by (2.1), where $\epsilon>0$ is chosen so that $\operatorname{Ric}_{d t^{2}+g(t)}>0$. Then there exists small enough $\tau<<\epsilon$ and a $C^{2}$-metric $d t^{2}+\tilde{g}(t)$ such that

(i) $\operatorname{Ric}_{d t^{2}+\tilde{g}(t)}>0$;

(ii) $\tilde{g}(t)=g(t)$ if $|t| \geq \tau$;

(iii) the metrics $d t^{2}+\tilde{g}(t)$ and $d t^{2}+g(t)$ are arbitrarily $C^{1}$-close on $X \times[-\epsilon, \epsilon]$.

Proof of Lemma 2. Indeed, we choose a coordinate system on $X \times[-\epsilon, \epsilon]$, so that the metric $g(t)$ is given by its components $g_{i j}(t)$, which are smooth when $t \neq 0$ and $C^{1}$-functions when $t=0$. Then we use the polynomial 2.7) for each function $g_{i j}(t)$ to obtain functions $\tilde{g}_{i j}(t)$. Clearly by choosing $\tau$ sufficiently small we can bound the variation in the metric components $\tilde{g}_{i j}(t)$ and their first derivatives by an arbitrarily small constant, whereas the second derivatives vary between their 
values at the endpoints. As curvature is a $C^{2}$ phenomenon which depends linearly on the second derivatives of the metric, we see that any open convex curvature condition satisfied by both 'halves' of the $C^{1}$ metric (i.e. either side of $t= \pm \epsilon$ ) will continue to be satisfied by the resulting $C^{2}$ metric, as $\tau$ is chosen to be sufficiently small. Since the positivity of the Ricci curvature is an open and convex condition we deduce that the our $C^{2}$ metric $d t^{2}+\tilde{g}(t)$ will have positive Ricci curvature if $\tau$ is sufficiently small.

Proof of Theorem 2 continued. It remains to smooth the metric from $C^{2}$ to $C^{\infty}$. By general smoothing theory for functions, we know that the set of $C^{2}$ functions on a smooth manifold is dense in the space of $C^{\infty}$ functions (see for Theorem 2.6 of [15]). Thus we can make a $C^{2}$-arbitrarily small adjustment to our $C^{2}$ metric to render it smooth, and in so doing ensure the positivity of the Ricci curvature is preserved. This proves Theorem 2 .

Theorem 2 immediately gives us the following corollary, which will play a key role in section 4 .

Corollary 1. The conclusion of Theorem 2 holds if the normal curvatures at both boundaries (with respect to the outward normals) are all positive.

2.3. A family version of Perelman's Theorem. We will also need a family version of Theorem 2, which allows us to perform simulataneous Ricci positive smoothings on the fibres of a bundle.

Theorem 3. Let $\pi_{i}: E_{i} \rightarrow B, i=1,2$ be smooth compact fibre bundles with fibre $M_{i}$, where $\partial M_{i} \neq \emptyset$. Suppose that each of these bundles is equipped with a smoothly varying family of fibrewise Ricci positive metrics $\left\{h_{i}(b)\right\}_{b \in B}$, and that with respect to these metrics, there is a smoothly varying family of fibrewise isometries $\phi:=\left\{\phi_{b}\right\}_{b \in B}$ for the boundary bundles $\partial \pi_{i}: \partial E_{i} \rightarrow B$ (with fibre $\left.\partial M_{i}\right)$, that is, $\phi_{b}: \partial \pi_{1}^{-1}(b) \cong \partial \pi_{2}^{-1}(b)$ for each $b \in B$. Then provided the normal curvatures of $\left.h_{1}(b)\right|_{\partial \pi_{1}^{-1}(b)}$ with respect to the outward normal are always greater than the negatives of corresponding normal curvatures of $\left.h_{2}(b)\right|_{\partial \pi_{2}^{-1}(b)}$ with respect to its outward normal, the fibrewise $C^{0}$-metric $h:=$ $\left\{h_{1}(b) \cup_{\phi(b)} h_{2}(b)\right\}_{b \in B}$ on $E_{1} \cup_{\phi} E_{2}$ can be smoothed within fibrewise positive Ricci curvature in such a way that the resulting metric agrees with the original ouside a neighbourhood of the glued boundaries.

Proof. The key observation is that in the proof of Theorem 2 , the $C^{2}$-smoothing constructed there only depends on the metrics together with two small positive parameters $\epsilon$ and $\tau$. Now suppose we have a smooth variation of the metrics on $M_{1}$ and $M_{2}$, which nevertheless always satisfies the requirements of Theorem 2. It is clear that $\epsilon$, the first chosen parameter in the construction which together with the given metrics determines the $C^{1}$ smoothing, can be chosen to vary continuously with the metric. Similarly the second parameter, $\tau$, needed to construct the $C^{2}$ smoothing, can be chosen to vary continuously with the metrics and $\epsilon$.

In the situation of the current Theorem, it follows from the above observations and the compactness of $B$ that we can make uniform choices for $\epsilon$ and $\tau$ which will work for all fibres in our bundles $E_{1}$ and $E_{2}$. Having made these choices, the $C^{2}$ metric smoothing performed after gluing each pair of fibres is then completely determined by the metrics on these fibres. Moreover, since this 
is a smoothing by polynomials, it follows trivially that the resulting metrics will vary smoothly from fibre to fibre.

Finally, the same argument as employed at the end of the proof of Theorem 2 shows that our fibrewise Ricci positive metric on $E_{1} \cup_{\phi} E_{2}$ can be smoothed to class $C^{\infty}$ within fibrewise positive Ricci curvature. (We could always extend our fibrewise $C^{2}$ metric to a global $C^{2}$ metric for which the intrinsic fibre metrics have positive Ricci curvature. This can then be globally smoothed by a $C^{2}$-arbitrarily small deformation, preserving the intrinsic positive Ricci curvature on the fibres, then restricting to the fibres yields the desired smooth fibrewise metric.)

\section{HATChER BUNDLES}

3.1. Goette's Theorem. The aim of this section is to review the construction and properties of certain smooth $S^{n}$-bundles over $S^{i}$ known as "Hatcher bundles". In short, a Hatcher bundle $E_{\lambda} \rightarrow S^{i}$ is a smooth $S^{n}$-bundle determined by an element $\lambda \in \operatorname{ker} J$, where $J: \pi_{i-1} O(p) \longrightarrow \pi_{i-1+p} S^{p}$ is the $J$-homomorphism, and where $0<p<n$. A Hatcher bundle $E_{\lambda} \rightarrow S^{i}$ has structure group $\operatorname{Diff}_{x_{0}}\left(S^{n}\right)$ and thus is classified by some map $f_{\lambda}: S^{i} \rightarrow \operatorname{BDiff}_{x_{0}}\left(S^{n}\right)$. We then say that a Hatcher bundle $E_{\lambda} \rightarrow S^{i}$ represents the element $\left[f_{\lambda}\right] \in \pi_{i} \operatorname{BDiff}_{x_{0}}\left(S^{n}\right)$; below we identify BDiff $x_{0}\left(S^{n}\right)=\mathcal{M}_{x_{0}}\left(S^{n}\right)$.

In the introduction we stated a theorem of Farrell and Hsiang (Theorem 1) concerning the rational homotopy groups $\pi_{i} \mathcal{M}_{x_{0}}\left(S^{n}\right) \otimes \mathbb{Q}$. In fact, each element of those groups may be represented by a Hatcher bundle.

Theorem 4. (See [12, Section 5]) Suppose that $n$ and $k$ satisfy the hypotheses of Theorem 1, so $\pi_{4 k} \mathcal{M}_{x_{0}}\left(S^{n}\right) \otimes \mathbb{Q} \cong \mathbb{Q}$ for such $k$ and $n$. Then for each element $[f] \in \pi_{4 k} \mathcal{M}_{x_{0}}\left(S^{n}\right) \otimes \mathbb{Q}$, there is an integer $p$ with $0<p<n$, and an element $\lambda \in \operatorname{ker} J$, where $J: \pi_{i-1} O(p) \longrightarrow \pi_{i-1+p} S^{p}$ is the $J$-homomorphism, such that the Hatcher bundle $E_{\lambda}$ represents the element $[f]$.

We recall that the key feature of these bundles is that they are exotic smooth $S^{n}$-bundles, in the sense that each one is homeomorphic to, but not diffeomorphic to, the trivial bundle $S^{i} \times S^{n} \rightarrow S^{i}$. We will develop Goette's construction so as to provide the appropriate setting for our geometric arguments in the next section. An in-depth description of these bundles and their properties is given in [12], and we refer the reader to this paper for further details.

3.2. Preliminary constructions. Throughout this section, we will assume that $n$ is odd and is sufficiently large for all of our purposes. The groups $\pi_{i} \mathcal{M}_{x_{0}}\left(S^{n}\right) \otimes \mathbb{Q}$ are trivial unless $i=4 k$ for appropriate $k$, and so we will consider only bundles which have base manifold $S^{4 k}$ and fibre $S^{n}$.

Let us begin with the trivial bundle $S^{4 k} \times S^{n} \rightarrow S^{4 k}$. By decomposing the fibre sphere $S^{n}$ into a pair of northern and southern hemispherical discs, $D_{+}^{n}$ and $D_{-}^{n}$, we can decompose the entire bundle into a pair of disc bundles, $S^{4 k} \times D_{+}^{n} \rightarrow S^{4 k}$ and $S^{4 k} \times D_{-}^{n} \rightarrow S^{4 k}$, glued together in the obvious way. Thus the trivial bundle $S^{4 k} \times S^{n} \rightarrow S^{4 k}$ can be regarded as the double of the trivial disc bundle $S^{4 k} \times D^{n} \rightarrow S^{4 k}$. We will always assume that discs are closed unless otherwise stated.

To construct a Hatcher bundle, we will make certain adjustments to the trivial $D^{n}$-bundle over $S^{4 k}$ to obtain a smooth bundle which is homeomorphic to, but not diffeomorphic to the trivial 
disc bundle. We will then form the double of this exotic $D^{n}$-bundle to obtain the desired exotic $S^{n}$-bundle over $S^{4 k}$, which will represent a non-trivial element of $\pi_{4 k} \mathcal{M}_{x_{0}}\left(S^{n}\right) \otimes \mathbb{Q}$.

We begin with the trivial disc bundle $S^{4 k} \times D^{n} \rightarrow S^{4 k}$. The fibre $D^{n}$ decomposes as

$$
\begin{aligned}
D^{n} & =D^{p+1} \times D^{q} \\
& \left.=\left(D^{p+1}(\rho) \times D^{q}\right) \cup\left(S^{p} \times[\rho, 1]\right) \times D^{q}\right),
\end{aligned}
$$

where $p+q+1=n, \rho \in(0,1)$, and $D^{p+1}(\rho) \times D^{q}$ is a smaller version of the original disc product (with the $D^{p+1}$ factor having radius $\rho$ ) surrounded by an annular region $\left(S^{p} \times[\rho, 1]\right) \times D^{q}$. The integers $p$ and $q$ may be assumed to be positive; in fact at various stages in the construction, it is necessary to allow both $p$ and $q$ to be large. It will be convenient for later considerations to reorder the factors and write the annular region as $S^{p} \times D^{q} \times[\rho, 1]$. Henceforth we will denote this by $A$ and the remaining piece, $D^{p+1}(\rho) \times D^{q}$, by $P{ }^{2}$ Thus, as illustrated in Fig. 3 below, we have

$$
D^{n}=A \cup P .
$$

The regions, $A$ and $P$, share a common piece of boundary, $S^{p} \times D^{q} \times\{\rho\}$, and are glued together
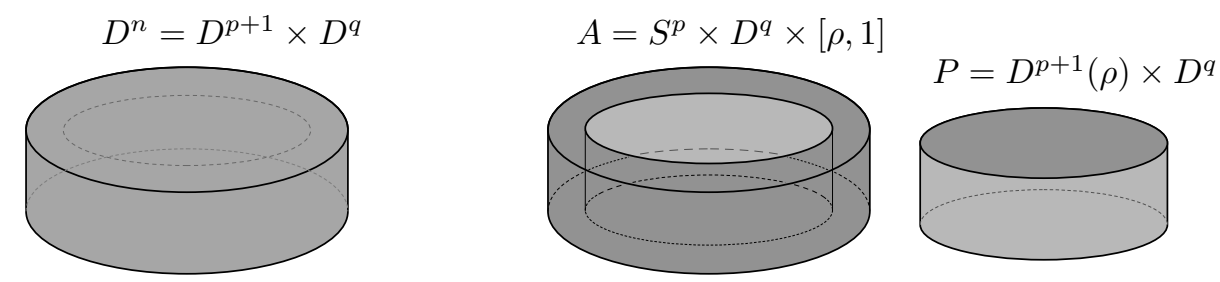

Figure 3. The decomposition of the fibre disc $D^{n}$ into $A$ and $P$

via the identity map on $S^{p} \times D^{q}$. The trivial bundle $S^{4 k} \times D^{n} \rightarrow S^{4 k}$, therefore, can be thought of as a union of sub-bundles $S^{4 k} \times A \rightarrow S^{4 k}$ and $S^{4 k} \times P \rightarrow S^{4 k}$, glued together in the obvious way.

From now on we will write $A_{y}=\{y\} \times A$ and $P_{y}=\{y\} \times P$, to denote the fibres at $y \in S^{4 k}$ of the respective sub-bundles $S^{4 k} \times A \rightarrow S^{4 k}$ and $S^{4 k} \times P \rightarrow S^{4 k}$. Below we will specify a smooth diffeomorphism

$$
\Lambda_{y}: S^{p} \times D^{q} \rightarrow S^{p} \times D^{q}
$$

over each $y \in S^{4 k}$, where the domain is $\left(\partial D^{p+1}\right) \times D^{q} \subset \partial P_{y}$ and the target space is the product $S^{p} \times D^{q} \times\{\rho\} \subset \partial A_{y}$. The idea will be to replace the identity map on $S^{p} \times D^{q}$, which glues $P_{y}$ to $A_{y}$ to form $D^{p+1} \times D^{q}$, with the map $\Lambda_{y}$.

Before we can begin the construction we will need a further decomposition: that of the base manifold $S^{4 k}$ into northern and southern hemispherical discs

$$
S^{4 k}=D_{+}^{4 k} \cup D_{-}^{4 k} .
$$

Over the disc $D_{-}^{4 k}$ we take the trivial bundle $D_{-}^{4 k} \times D^{n} \rightarrow D_{-}^{4 k}$, that is, we define the map $\Lambda_{y}$ to be the identity map on $S^{p} \times D^{q}$ for all $y \in D_{-}^{4 k}$. We therefore need to specify the maps $\Lambda_{y}$ for $y \in D_{+}^{4 k}$

2 This smaller product of discs resembles an ice-hockey puck, hence the notation. 
in order to describe the bundle over $D_{+}^{4 k}$, and finally we need to show how to glue the two bundles together over $S^{4 k-1}=\partial D_{-}^{4 k}=\partial D_{+}^{4 k}$.

Over the disc $D_{+}^{4 k}$ we will actually work with a slightly different, though topologically equivalent annulus $\mathcal{A}_{y}$, which we will define below. We will also work with diffeomorphisms $\Lambda_{y}$ as above, however we must adjust the target space to lie in the boundary of $\mathcal{A}_{y}$. Gluing the $P_{y}$ to $\mathcal{A}_{y}$ creates a fibre bundle over $D_{+}^{4 k}$ with fibres $P_{y} \cup_{\Lambda_{y}} \mathcal{A}_{y}$.

In order to make these constructions, let us first suppose we have a collection of embeddings

$$
\bar{\lambda}_{y}: S^{p} \times D^{q} \longrightarrow S^{p} \times D^{q}
$$

for $y \in D_{+}^{4 k}$ which vary smoothly with $y$. For any given $y \in D_{+}^{4 k}$, the image of the embedding $\bar{\lambda}_{y}$,
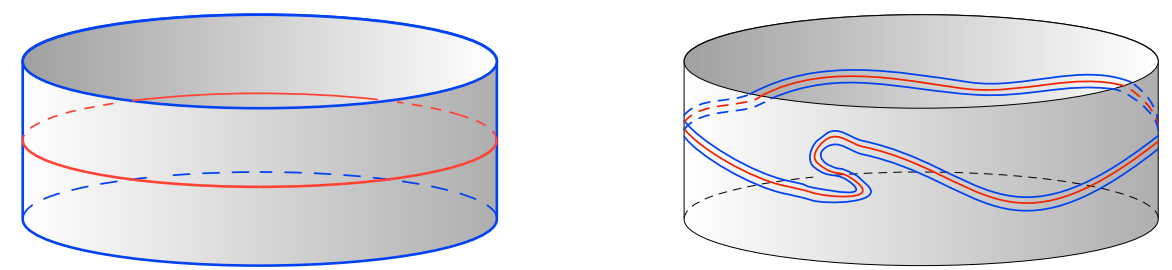

Figure 4. An embedding $\bar{\lambda}_{y}$ from $S^{p} \times D^{q}$ (left) into $S^{p} \times D^{q}$ (right)

which we denote $\operatorname{Im} \bar{\lambda}_{y}$, is schematically depicted in Fig. 4. We will define the annulus $\mathcal{A}_{y}$ by

$$
\mathcal{A}_{y}=\{y\} \times \operatorname{Im} \bar{\lambda}_{y} \times[\rho, 1] \subset\{y\} \times S^{p} \times D^{q} \times[\rho, 1] .
$$

We will furthermore define the diffeomorphism $\Lambda_{y}$ to be simply the map $\bar{\lambda}_{y}$ with target space $\operatorname{Im} \bar{\lambda}_{y}$. Thus we can glue $P_{y}$ to $\mathcal{A}_{y}$ using $\Lambda_{y}$, by identifying the points

$$
(y, x) \in\{y\} \times\left(S^{p} \times D^{q}\right) \subset \partial P_{y} \quad \text { and } \quad\left(y, \bar{\lambda}_{y}(x), \rho\right) \in\{y\} \times \operatorname{Im} \bar{\lambda}_{y} \times\{\rho\} \subset \partial \mathcal{A}_{y},
$$

where $x \in S^{p} \times D^{q}$. The spaces $P_{y}$ and $\mathcal{A}_{y}$ (as a subset of $\{y\} \times S^{p} \times D^{q} \times[\rho, 1]$ ) are depicted in Fig. 5 below. Applying this gluing fibrewise for all $y \in D_{+}^{4 k}$ gives rise to the desired bundle over $D_{+}^{4 k}$. For convenience we will denote the bundles over $D_{ \pm}^{4 k}$ by $\mathcal{E}_{ \pm} \rightarrow D_{ \pm}^{4 k}$. We claim that for each
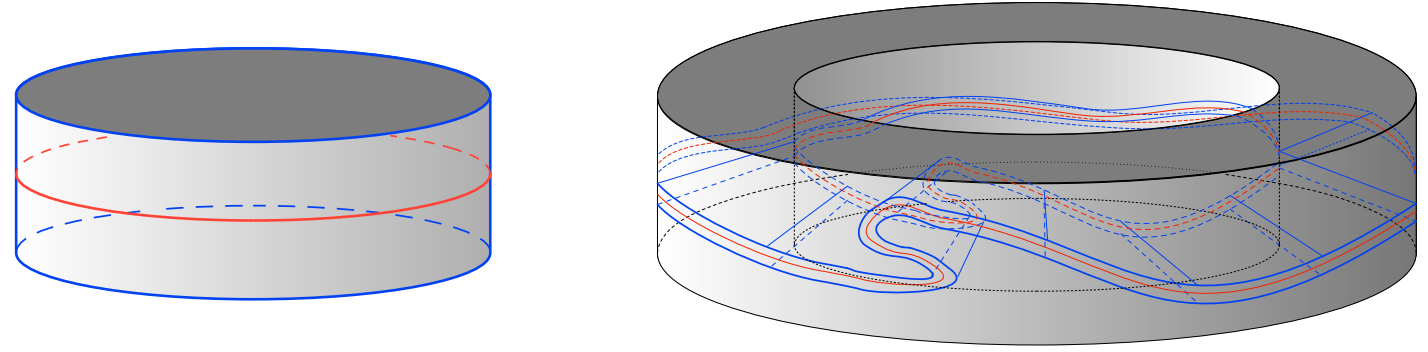

Figure 5. The spaces $P_{y}$ (left) and $\mathcal{A}_{y}$, as a subset of $S^{p} \times D^{q} \times[\rho, 1]$ (right) 
$y \in D_{+}^{4 k}$ we have a canonical diffeomorphism

$$
D^{p+1} \times D^{q}=P_{y} \cup_{I d} A_{y} \cong P_{y} \cup_{\Lambda_{y}} \mathcal{A}_{y} .
$$

To this end, define a map $\phi_{y}: A_{y} \rightarrow \mathcal{A}_{y}$ by setting $\phi_{y}=\Lambda_{y} \times I d_{[\rho, 1]}$, where we are viewing $A_{y}=\left(S^{p} \times D^{q}\right) \times[\rho, 1]$ and $\mathcal{A}_{y}=\operatorname{Im} \bar{\lambda}_{y} \times[\rho, 1]$. Using this map we can define a further map $\Phi_{y}: P_{y} \cup_{I d} A_{y} \rightarrow P_{y} \cup_{\Lambda_{y}} \mathcal{A}_{y}$ by

$$
\Phi_{y}(z)= \begin{cases}z & z \in P_{y} \\ \phi_{y}(z) & z \in A_{y} .\end{cases}
$$

It is clear that $\Phi_{y}$ is a homeomorphism for each $y$. Following the discussion on adjunction spaces at the start of section 2 (or see [15, Chapter 8, section 2]), we see that with respect to the canonical differentiable structure on $P_{y} \cup_{\Lambda_{y}} \mathcal{A}_{y}, \Phi_{y}$ is actually a diffeomorphism. Thus the exotic structure which Hatcher bundles display does not occur at the level of individual fibres: it is a global bundle phenomenon.

3.3. Recollection of the $J$-homomorphism. Here we follow [12] to construct the family of embeddings $\bar{\lambda}_{y}: S^{p} \times D^{q+1} \rightarrow S^{p} \times D^{q+1}$, for $y \in D_{+}^{4 k}$, as above. This requires us to consider the $J$-homomorphism, which is a map

$$
J: \pi_{4 k-1} O(p) \longrightarrow \pi_{4 k-1+p} S^{p},
$$

where $p$ is sufficiently large $3^{3}$ We can think of the $J$-homomorphism as follows. Consider a map $\lambda: S^{4 k-1} \rightarrow O(p)$ determined by a choice of element $[\lambda] \in \pi_{4 k-1} O(p)$; by the Whitney approximation theorem, without loss of generality we can assume that $\lambda$ is smooth. This then determines a map $S^{4 k-1} \times \mathbb{R}^{p} \rightarrow \mathbb{R}^{p}$, by sending a point $(y, z)$ to the orthogonal transformation $\lambda(y) \in O(p)$ applied to $z \in \mathbb{R}^{p}$. Since $S^{p}=\mathbb{R}^{p} \cup\left\{x_{0}\right\}$, it is convenient to identify $\lambda$ with a map $\lambda: S^{4 k-1} \rightarrow \mathcal{C}\left(S^{p}, S^{p}\right)$, where $\mathcal{C}(X, Y)$ is a space (with compact-open topology) of continuous maps $X \rightarrow Y$ preserving basepoints. It will also be convenient to denote by $\lambda_{y}: S^{p} \rightarrow S^{p}$, the map $\lambda$ evaluated at $y \in S^{4 k-1}$. Passing to homotopy classes gives a map $\pi_{4 k-1} O(p) \rightarrow \pi_{4 k-1} \mathcal{C}\left(S^{p}, S^{p}\right)$, and composing this with an isomorphism $\pi_{4 k-1} \mathcal{C}\left(S^{p}, S^{p}\right) \cong \pi_{4 k-1+p} S^{p}$ then gives the $J$-homomorphism.

Recall that for $p$ sufficiently large compared to $k$, the groups $\pi_{4 k-1} O(p)$ and $\pi_{4 k-1+p} S^{p}$ are independent of $p$, and $\pi_{4 k-1+p} S^{p}$ is a finite group, while $\pi_{4 k-1} O(p)$ is infinite cyclic.

Choose a map $\lambda: S^{4 k-1} \rightarrow O(p)$ such that $[\lambda] \neq 0$ and $[\lambda] \in$ ker $J$. This means that $\lambda$ extends to a map

$$
\tilde{\lambda}: D_{+}^{4 k} \rightarrow \mathcal{C}\left(S^{p}, S^{p}\right)
$$

where $D_{+}^{4 k}$ is a disc of radius 1 . We can assume that $\tilde{\lambda}$ restricted to the collar $S^{4 k-1} \times(1 / 2,1] \subset D_{+}^{4 k}$ coincides with the product-map $\lambda \times \operatorname{Id}$. For any $q \geq 4 k$, we denote by $\iota: S^{p} \rightarrow S^{p} \times D^{q}$ the inclusion

$\overline{3}$ Here we assume that a base point in $S^{p}$ is the north pole. 
$\iota: x \mapsto(x, 0)$. The map $\lambda$ and its extension $\tilde{\lambda}$ give a commutative diagram

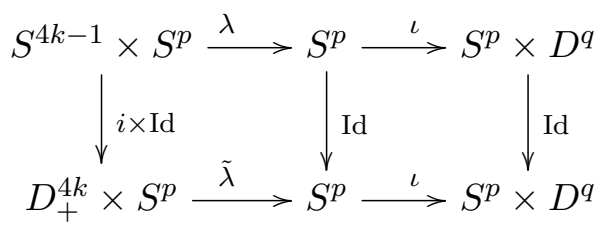

where $i$ is the inclusion of the boundary $S^{4 k-1} \rightarrow D_{+}^{4 k}$.

Lemma 3. For sufficiently large $q$, we can approximate the map $\iota \circ \tilde{\lambda}$ by a smoothly varying family of smooth embeddings

$$
\hat{\lambda}_{y}: S^{p} \longrightarrow S^{p} \times D^{q}, \quad y \in D_{+}^{4 k},
$$

which retain the property that for $y \in S^{4 k-1} \times(1 / 2,1] \subset D_{+}^{4 k}$, the maps $\hat{\lambda}_{y}$ agree with $\iota \circ \lambda_{y}$.

Proof. We begin by recalling that the map $\lambda: S^{4 k-1} \rightarrow O(p)$ is assumed to be smooth. By applying the Whitney approximation theorem to $\tilde{\lambda}$ viewed as a map $\tilde{\lambda}: D_{+}^{4 k} \times S^{p} \rightarrow S^{p}$, (see for example [18, Theorem 6.19]), we see that by a $C^{0}$ arbitrarily small homotopy relative to a boundary neighbourhood in the domain, we can adjust this map to be smooth. Thus without loss of generality, we may as well assume in the first place that $\tilde{\lambda}: D_{+}^{4 k} \times S^{p} \rightarrow S^{p}$ is smooth.

To construct the embeddings $\hat{\lambda}_{y}: S^{p} \rightarrow S^{p} \times D^{q}$, we first let $\eta: S^{p} \rightarrow D^{q}$ be an arbitrary embedding of the sphere into a ball $D^{q} \subset \mathbb{R}^{q}$ centered at the origin, and denote by $\epsilon \eta$ the composition consisting of $\eta$ followed by a scaling of $D^{q}$ onto itself by a factor of $\epsilon \geq 0$. (The embedding $\eta$ clearly exists provided that $q \geq p+1$.) Next, we introduce a function $\epsilon: D_{+}^{4 k} \rightarrow \mathbb{R}$, which is identically zero in a small neighbourhood of the boundary of $D_{+}^{4 k}$ within the region in which $\tilde{\lambda}$ is independent of the radial parameter, is strictly positive otherwise, and is everywhere smooth.

Finally, set $\hat{\lambda}: D_{+}^{4 k} \times S^{p} \rightarrow S^{p} \times D^{q}$ to be the map

$$
\hat{\lambda}(y, x) \mapsto\left(\tilde{\lambda}_{y}(x), \epsilon(y) \eta(x)\right) .
$$

It is now immediate that this restricts to give a smoothly varying family of smooth embeddings $\hat{\lambda}_{y}: S^{p} \longrightarrow S^{p} \times D^{q}$, by virtue of the fact that $\eta$ is an embedding. Moreover, these embeddings clearly agree with $\iota \circ \tilde{\lambda}_{y}$ for $y$ close to the boundary of $D_{+}^{4 k}$, since $\epsilon$ vanishes in this region.

Denote by $N_{y} \rightarrow S^{p}$ the normal bundle of the embedding $\hat{\lambda}_{y}$. Considering all $y \in D_{+}^{4 k}$, we obtain a vector bundle $N \rightarrow D_{+}^{4 k} \times S^{p}$. Since $N_{y} \rightarrow S^{p}$ is a trivial bundle, the bundle $N \rightarrow D_{+}^{4 k} \times S^{p}$ is also trivial. Then by fixing a trivialization of $N$ and using the normal exponential map, we extend the family of embeddings (3.4) to the family of embeddings

$$
\bar{\lambda}_{y}: S^{p} \times D^{q} \longrightarrow S^{p} \times D^{q}, \quad y \in D_{+}^{4 k} .
$$

Lemma 4. [12, Proposition 5.4] The family of embeddings (3.5) is smoothly isotopic to a family of embeddings $S^{p} \times D^{p} \longrightarrow S^{p} \times D^{q}$ which restricts over $S^{4 k-1}=\partial D_{+}^{4 k}$ to give linear transformations

$$
(x, z) \mapsto\left(\lambda_{y}(x),\left(\lambda_{y}^{-1} \oplus \operatorname{Id}_{\mathbb{R}^{p-q}}\right)(z)\right) \in S^{p} \times D^{q}, \quad y \in S^{4 k-1} .
$$


Remark. According to Lemma 4, we can assume that the family of embeddings (3.5) satisfies the condition (3.6). We notice also that (3.6) implies that $\operatorname{Im} \bar{\lambda}_{y}$ coincides with $\{y\} \times S^{p} \times D^{q} \subset \partial A_{y}$. In particular, we can assume that

$$
\mathcal{A}_{y}=A_{y} \quad \text { if } y \in D_{+}^{4 k} \text { is near the boundary } \partial D_{+}^{4 k} .
$$

Now recall that the embeddings $\bar{\lambda}_{y}$ give rise to the desired family of diffeomorphisms $\Lambda_{y}$, which by definition coincide with the maps $\bar{\lambda}_{y}$ when the target space is restricted to $\operatorname{Im} \bar{\lambda}_{y}$. This completes the construction of the bundle $\mathcal{E}_{+} \rightarrow D_{+}^{4 k}$.

We conclude this section with the result below, which follows from the proof of Lemma 3

Corollary 2. The diffeomorphisms $\Lambda_{y}: S^{p} \times D^{q} \rightarrow S^{p} \times D^{q}$ are determined by their restriction to an arbitrarily small neighbourhood of the sphere $S^{p} \times\{0\} \subset S^{p} \times D^{q} \subset \partial P_{y}$ and its image in $\partial \mathcal{A}_{y}$, for each $y \in D_{+}^{4 k}$.

Proof. We merely have to observe that in the proof of Lemma 3 we can choose the embedding $\eta$ so that its image is contained in an arbitrarily small ball about the origin in $D^{q}$, and we can choose the function $\epsilon$ to have an arbitrarily small upper bound.

3.4. The Hatcher bundle $E_{\lambda}$. It remains to describe how the bundle $\mathcal{E}_{+} \rightarrow D_{+}^{4 k}$ is to be glued to the trivial disc bundle $\mathcal{E}_{-} \rightarrow D_{-}^{4 k}$ along the boundary of the base discs $\partial D_{+}^{4 k}=\partial D_{-}^{4 k}=S^{4 k-1}$. Recall that each disc fibre is the union of an annulus and a 'puck', and, according to (3.7), we can assume the annulus parts $\mathcal{A}_{y}$ of the fibres are equal to $A_{y}$ near the boundary of $D_{+}^{4 k}$.

We can therefore begin by gluing the annulus parts, $A_{y}$ and $\mathcal{A}_{y}$, of the fibres at the boundary of $\mathcal{E}_{+}$and $\mathcal{E}_{-}$via the identity map. To glue the 'puck' part of the fibres we observe that, according to Lemma 4, the maps $\Lambda_{y}: S^{p} \times D^{q} \rightarrow S^{p} \times D^{q}$ defined above are products of rotations for each $y$ near to $\partial D_{+}^{4 k}$. Thus the $\Lambda_{y}$ extend to diffeomorphisms $\tilde{\Lambda}_{y}: P_{y} \rightarrow P_{y}$ for such $y$, using the rotations (3.6). We use the $\tilde{\Lambda}_{y}$ to glue the puck sub-bundles of $\mathcal{E}_{+}$to $\mathcal{E}_{-}$for $y \in S^{4 k-1}$, noting that this is consistent with the gluing of the annuli. The disc bundle over $S^{4 k}$ which results from this gluing we will denote by $\mathcal{E}_{\lambda}$, since it ultimately depends on our choice of $[\lambda] \in \operatorname{ker} J$. As noted at the start of this section, we can then double this disc bundle to produce a desired Hatcher bundle $E_{\lambda}:=\mathcal{E}_{\lambda} \cup \mathcal{E}_{\lambda}$ over $S^{4 k}$.

It can be shown that $E_{\lambda}$ is bundle homeomorphic but not bundle diffeomorphic to the corresponding trivial bundle, see [12, Proposition 5.8, Theorem 5.13] and [14, Section 1].

\section{The Fibrewise Ricci positive metric Construction}

4.1. Foreword. In this section we will ultimately prove Main Theorem. As discussed in section 1. this reduces to showing that a Hatcher bundle admits a fiberwise Ricci positive metric. Our general strategy is to show the existence of fiberwise Ricci positive metrics on the Hatcher disc bundles constructed in the last section, and then use the family version of the Perelman gluing result, Theorem 3, to glue two copies of such a disc bundle together within Ricci positivity to create 
the desired object. In order to perform this gluing, we need to consider the normal curvatures at the boundary of the disc fibres.

There is an immediate problem, however, with the boundary of the discs: these were constructed as products $D^{p+1} \times D^{q}$. Thus, as written, each of these is a manifold with corners. Moreover, in order for these discs to be equipped with Ricci positive metrics, it is natural to consider product metrics which respect the topological product structure. The resulting boundary is not smooth, however, and we need a smooth boundary in order to apply the Perelman gluing technique.

In order to deal with this issue, our approach is to cut out a solid 'ellipsoid' from within the product of discs; see Fig. 6. This will be constructed to have a smooth boundary and normal curvatures at the boundary (with respect to the outward normal) which are all positive. Thus, provided the ambient metric on the product of discs has positive Ricci curvature, we can glue two such ellipsoids together using the Perelman gluing technique. Our main task in this section is therefore to show how to construct such an ellipsoid, and prove that it has the desired properties.

To avoid any confusion with indices, it is convenient to work with the product $D^{m} \times D^{n}$, where the role of $m$ and $n$ is symmetric. We specify the formulas for $m=p+1, n=q$ at the end of the section when we will prove Main Theorem.

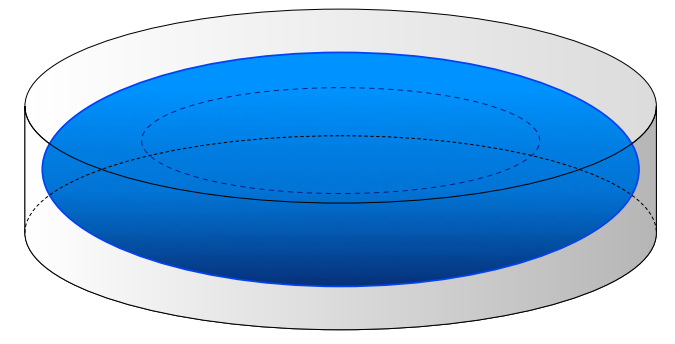

Figure 6 . The solid ellipsoid contained in $D^{m} \times D^{n}$

4.2. The metric on $D^{m} \times D^{n}$. First, we will consider the following metric on $D^{m} \times D^{n}$ :

$$
h:=d s^{2}+\alpha^{2}(s) d s_{m-1}^{2}+d t^{2}+\beta^{2}(t) d s_{n-1}^{2},
$$

where $s$ and $t$ are the radial parameters in the discs $D^{m}$ and $D^{n}$ respectively. (In our later metric constructions we will use a slight variant of this metric.) Let us assume that the radii of the two discs are $s_{1}$ and $t_{1}$ respectively. We will impose the following conditions on the smooth warping functions $\alpha$ and $\beta$ :

- $\alpha, \beta$ are odd in a small neighbourhood of $s=0$ respectively $t=0$ (or rather, one can extend $\alpha$ and $\beta$ to negative values of $s$ and $t$ such that this extended function is smooth and odd), and in particular $\alpha(0)=\beta(0)=0$;

- $\alpha^{\prime}(0)=\beta^{\prime}(0)=1$;

- $\alpha^{\prime}>0$ and $\beta^{\prime}>0$ whenever $s$ respectively $t$ is positive;

- $\alpha^{\prime \prime}(s)<0$ for all $s \in\left[0, s_{1}\right]$ and $\beta^{\prime \prime}(t)<0$ for all $t \in\left[0, t_{1}\right]$. 


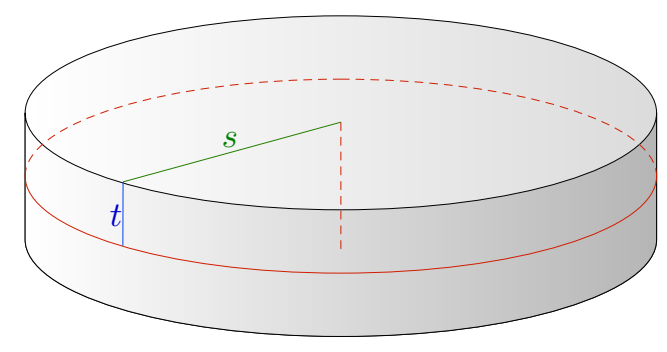

Figure 7. Coordinates on the space $X=D^{m} \times D^{n}$

It follows easily from the warped product formulas for Ricci curvature that these conditions ensure that the metric $h$ has strictly positive Ricci curvature, see, for example, [1, section 9J].

4.3. Specifying the ellipsoid. In order to construct the ellipsoid, we introduce a unit speed curve $\mu=\mu(r)$ into the $(s-t)$-plane. This curve will have the profile given in Fig. 8

Notice that the illustrated curve separates the rectangle $\left[0, s_{1}\right] \times\left[0, t_{1}\right]$ into two regions, and suppose that the parameter $r$ is such that $\mu(0)=\left(0, t_{0}\right)$ and $\mu\left(r_{0}\right)=\left(s_{0}, 0\right)$ for some $s_{0} \in\left(0, s_{1}\right)$ and $t_{0} \in\left(0, t_{1}\right)$. We will define the ellipsoid $\mathbf{E}$ to be the subset of $D^{p+1} \times D^{q}$ consisting of all elements whose $s$ and $t$ coordinates lie in the region on or below this curve.

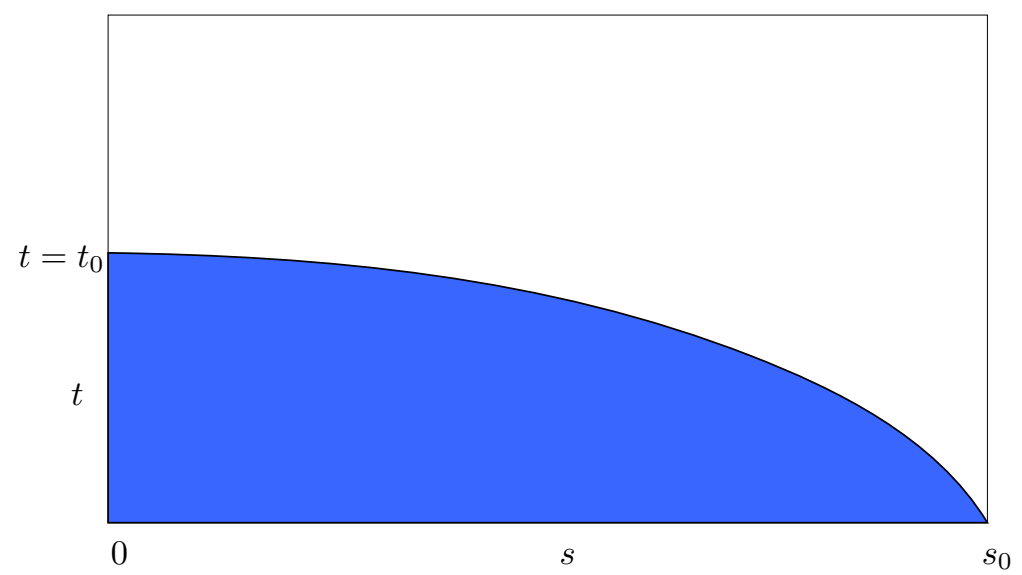

Figure 8. The curve $\mu$ which gives rise to the ellipsoid $\mathbf{E}$

We need to specify $\mu$ in more detail, and to this end we will write $\mu(r)=\left(\mu_{s}(r), \mu_{t}(r)\right)$. Let us impose the following conditions on $\mu_{s}, \mu_{t}$ :

(1) $\mu_{s}(0)=0, \mu_{s}\left(r_{0}\right)=s_{0}, \mu_{s}^{\prime}(0)=1, \mu_{s}^{\prime}\left(r_{0}\right)=0, \mu_{s}^{\prime \prime}(r)<0$ for all $r \in\left[0, r_{0}\right], \mu_{s}$ is odd in a neighbourhood of $r=0$, and even locally about the point $r=r_{0}$ (in the sense that there is a smooth extension such that $\mu_{s}\left(r_{0}-\epsilon\right)=\mu_{s}\left(r_{0}+\epsilon\right)$ for all sufficiently small $\left.\epsilon>0\right)$;

(2) $\mu_{t}(0)=t_{0}, \mu_{t}\left(r_{0}\right)=0, \mu_{t}^{\prime}(0)=0, \mu_{t}^{\prime}\left(r_{0}\right)=-1, \mu_{t}^{\prime \prime}(r)<0$ for all $r \in\left[0, r_{0}\right]$, $\mu_{t}$ is even about the point $r=0$, and odd in a neighbourhood of $r=r_{0}$. 
Given a unit speed curve $\mu$ satisfying (1) and (2) above, we need to check that the resulting ellipsoid has a smooth boundary. Firstly, it is clear from the smoothness of all the functions involved that this boundary will indeed be smooth everywhere except possibly when $r=0$ or $r=r_{0}$. We must therefore check the corresponding 'ends' of the ellipsoid for smoothness. With this in mind, we begin by observing that the metric induced by $h$ on the ellipsoid is

$$
h_{\mathbf{E}}:=d r^{2}+\alpha^{2}\left(\mu_{s}(r)\right) d s_{m-1}^{2}+\beta^{2}\left(\mu_{t}(r)\right) d s_{n-1}^{2} .
$$

Given that $\mu_{s}(0)=0, \mu_{t}\left(r_{0}\right)=0$, and that $\mu_{s}, \mu_{t}>0$ otherwise, we see immediately from the form of the metric, $h_{\mathbf{E}}$, that this is a (not necessarily smooth) metric on a sphere of dimension $m+n-1$. The boundary conditions which such a metric must satisfy in order to give a smooth sphere metric are well-known (see for example [20, Section 1.4]): the scaling funtions $\alpha\left(\mu_{s}(r)\right)$ and $\beta\left(\mu_{t}(r)\right)$ must obey the following rules

(i) be everywhere non-negative, with $\alpha\left(\mu_{s}(r)\right)=0$ if and only if $r=0$, and $\beta\left(\mu_{t}(r)\right)=0$ if and only if $r=r_{0}$;

(ii) $\alpha\left(\mu_{s}(r)\right)$ must be odd at $r=0$ and even at $r=r_{0}$;

(iii) $\beta\left(\mu_{t}(r)\right)$ must be even at $r=0$ and odd at $r=r_{0}$;

(iv) the derivative of $\alpha\left(\mu_{s}(r)\right)$ must take the value 1 at $r=0$, and that of $\beta\left(\mu_{t}(r)\right)$ must take the value -1 at $r=r_{0}$.

Property (i) follows immediately from the conditions imposed on $\alpha, \beta, \mu_{s}, \mu_{t}$. For property (iii) we note that by definition $\mu_{t}(r)$ is odd at $r=r_{0}$ and $\beta(t)$ is odd at $t=0$, and it follows trivially from this that the composition $\beta\left(\mu_{t}(r)\right)$ is odd at $r=r_{0}$. For the evenness requirement it suffices to note that the composition of an even function followed by an arbitrary function is trivially even. Property (ii) follows by similar arguments. Finally, property (iv) follows by the chain rule since $\alpha^{\prime}(0) \mu_{s}^{\prime}(0)=1$, and $\beta^{\prime}(0) \mu_{t}^{\prime}\left(r_{0}\right)=-1$.

In summary then, we have demonstrated how to choose a unit speed curve $\mu$ such that the resulting ellipsoid is smooth, and we will work with the same subset of $D^{p+1} \times D^{q}$ for each fibre of the Hatcher disc bundle when we construct the fiberwise metric later in this section.

4.4. Normal curvatures of the ellipsoid. The other issue we need to address in relation to the Perelman gluing of discs (or rather ellipsoids) is that of the normal curvatures at the boundary. As observed previously (Corollary 1), it will suffice if these normal curvatures are all positive with respect to the outward pointing normal. It turns out, however, that the normal curvatures of the ellipsoid we have constructed are only non-negative with respect to the ambient metric $h$. In particular, the normal curvatures vanish at the points of the ellipsoid corresponding to $r=0$ and $r=r_{0}$. To rectify this situation we work with the same ellipsoid, but a slightly modified metric on $D^{m} \times D^{n}$.

Let us define a metric $g$ on $D^{m} \times D^{n}$ as follows:

$$
g:=\delta^{2}(t) d s^{2}+\delta^{2}(t) \alpha^{2}(s) d s_{m-1}^{2}+\gamma^{2}(s) d t^{2}+\gamma^{2}(s) \beta^{2}(t) d s_{n-1}^{2}
$$

The new functions introduced here, $\delta(t)$ and $\gamma(s)$, are chosen so as to satisfy the following properties:

(a) $\delta^{\prime}(t) \geq 0$ for all $t \in\left[0, t_{1}\right], \delta^{\prime}\left(t_{0}\right)>0, \delta(t) \equiv 1$ in a neighbourhood of $t=0$; 
(b) $\gamma^{\prime}(s) \geq 0$ for all $s \in\left[0, s_{1}\right], \gamma^{\prime}\left(s_{0}\right)>0, \gamma(s) \equiv 1$ in a neighbourhood of $s=0$.

We will see that the positivity of the derivatives of $\delta$ and $\gamma$ at $t_{0}$, respectively $s_{0}$, is enough to give us strictly positive normal curvatures globally. Of course we must not forget that the metric $g$ must have positive Ricci curvature. By the openness of the positivity condition we can choose $\delta$ and $\gamma$ satisfying (a) and (b) above sufficiently close in a $C^{2}$-sense to the constant function with value 1 so that $\operatorname{Ric}(g)>0$. We therefore add a third condition:

(c) $\delta$ and $\gamma$ are such that $\operatorname{Ric}(g)>0$, at least in some neighbourhood of $\mathbf{E}$.

Lemma 5. The normal curvatures at the boundary of the ellipsoid $\mathbf{E}$ are all stricly positive with respect to the ambient metric $g$.

Proof. We work locally, and begin by fixing a point $x_{1}=\left(s_{1}, a_{1}, t_{1}, b_{1}\right) \in \mathbf{E}$, where $a_{1} \in S^{m-1}$ and $b_{1} \in S^{n-1}$. About the points $a_{1}$ and $b_{1}$, introduce normal coordinate systems locally into $S^{m-1}$ and $S^{n-1}$. Together with the $s$ and $t$ coordinates, these combine to give a local coordinate system in $D^{m} \times D^{n}$. With respect to these coordinates we can represent $g$ by the block-diagonal matrix

$$
g=\left[\begin{array}{cccc}
\delta^{2}(t) & & & \\
& \gamma^{2}(s) & & \\
& & \delta^{2}(t) \alpha^{2}(s) A_{m-1} & \\
& & & \gamma^{2}(s) \beta^{2}(t) B_{n-1}
\end{array}\right],
$$

where $A_{m-1}$ and $B_{n-1}$ represent $d s_{n-1}^{2}$ respectively $d s_{m-1}^{2}$ with respect to the chosen normal coordinate systems on the spheres. Note that at the the points $a_{1}$ and $b_{1}, A_{m-1}$ and $B_{n-1}$ are both identity matrices and have vanishing first derivatives. Hence at the point $x_{1}$ we have $g_{i j} \neq 0$ if and only if $i=j, g^{i i}=\frac{1}{g_{i i}}$, and the derivatives $g_{i j, k}=0$ whenever $k$ is a direction tangent to $S^{m-1}$ or $S^{n-1}$. We will assume that all computations below are carried out at this point.

Using the formula

$$
\Gamma_{i j}^{k}=\frac{1}{2} g^{k l}\left(g_{i l, j}+g_{j l, i}-g_{i j, l}\right),
$$

it is straightforward to compute the corresponding Christoffel symbols. The list below consists of precisely those Christoffel symbols which are non-zero. Beginning with the case when each of the indices $i, j$ and $k$ are $s$ or $t$, we have the following.

$$
\Gamma_{s t}^{s}=\Gamma_{t s}^{s}=\frac{\delta^{\prime}(t)}{\delta(t)}, \quad \Gamma_{t t}^{s}=\frac{-\gamma^{\prime}(s) \gamma(s)}{\delta^{2}(t)}, \quad \Gamma_{s s}^{t}=\frac{-\delta(t) \delta^{\prime}(t)}{\gamma^{2}(s)}, \quad \Gamma_{s t}^{t}=\Gamma_{t s}^{t}=\frac{\gamma^{\prime}(s)}{\gamma(s)} .
$$

Then, using the symbols $a$ and $b$ to represent any of the coordinate functions on $S^{m-1}$ or $S^{n-1}$ respectively, we list the remaining non-zero Christoffel symbols.

$$
\begin{gathered}
\Gamma_{a a}^{s}=-\alpha(s) \alpha^{\prime}(s), \quad \Gamma_{s a}^{a}=\Gamma_{a s}^{a}=\frac{\alpha^{\prime}(s)}{\alpha(s)}, \quad \Gamma_{a a}^{t}=\frac{-\delta(t) \delta^{\prime}(t) \alpha^{2}(s)}{\gamma^{2}(s)}, \quad \Gamma_{t a}^{a}=\Gamma_{a t}^{a}=\frac{\delta^{\prime}(t)}{\delta(t)} \\
\Gamma_{b b}^{s}=\frac{-\gamma(s) \gamma^{\prime}(s) \beta^{2}(t)}{\delta^{2}(t)}, \quad \Gamma_{s b}^{b}=\Gamma_{b s}^{b}=\frac{\gamma^{\prime}(s)}{\gamma(s)}, \quad \Gamma_{b b}^{t}=-\beta(t) \beta^{\prime}(t), \quad \Gamma_{t b}^{b}=\Gamma_{b t}^{b}=\frac{\beta^{\prime}(t)}{\beta(t)}
\end{gathered}
$$


From this we compute certain covariant derivatives involving coordinate vector fields, $\partial_{s}, \partial_{t}, \partial_{a}$ and $\partial_{b}$, which will we will make use of shortly. In particular, we see that at the point $x_{1}$ we have

$$
\begin{gathered}
\nabla_{\partial_{s}} \partial_{s}=\frac{-\delta(t) \delta^{\prime}(t)}{\gamma^{2}(s)} \partial_{t}, \quad \nabla_{\partial_{t}} \partial_{t}=\frac{-\gamma^{\prime}(s) \gamma(s)}{\delta^{2}(t)} \partial_{s}, \quad \nabla_{\partial_{s}} \partial_{t}=\nabla_{\partial_{t}} \partial_{s}=\frac{\delta^{\prime}(t)}{\delta(t)} \partial_{s}+\frac{\gamma^{\prime}(s)}{\gamma(s)} \partial_{t}, \\
\nabla_{\partial_{t}} \partial_{a}=\nabla_{\partial_{a}} \partial_{t}=\frac{\delta^{\prime}}{\delta} \partial_{a}, \quad \nabla_{\partial_{s}} \partial_{a}=\nabla_{\partial_{a}} \partial_{s}=\frac{\alpha^{\prime}}{\alpha} \partial_{a} \\
\nabla_{\partial_{t}} \partial_{b}=\nabla_{\partial_{b}} \partial_{t}=\frac{\beta^{\prime}}{\beta} \partial_{b}, \quad \nabla_{\partial_{s}} \partial_{b}=\nabla_{\partial_{b}} \partial_{s}=\frac{\gamma^{\prime}}{\gamma} \partial_{b}, \\
\nabla_{\partial_{a}} \partial_{a}=-\frac{\delta^{\prime} \delta \alpha^{2}}{\gamma^{2}} \partial_{t}-\alpha^{\prime} \alpha \partial_{s}, \quad \nabla_{\partial_{b}} \partial_{b}=-\frac{\gamma^{\prime} \gamma \beta^{2}}{\delta^{2}} \partial_{s}-\beta^{\prime} \beta \partial_{t},
\end{gathered}
$$

and $\nabla_{\partial_{a}} \partial_{b}=\nabla_{\partial_{b}} \partial_{a}=0$.

The statement that all normal curvatures are positive is clearly equivalent to the statement that the second fundamental form is positive definite. We will compute second fundamental forms, and will break up the computation into directions tangent to $S^{m-1}, S^{n-1}$, and tangent to the curve $\mu$. Notice that $\mu^{\prime}(r)$ is everywhere tangent to the boundary of the ellipsoid, and this direction is orthogonal (with respect to $g$ ) to both $S^{m-1}$ and $S^{n-1}$. Explicitly we have $T(r):=\mu^{\prime}(r)=\mu_{s}^{\prime}(r) \partial_{s}+\mu_{t}^{\prime}(r) \partial_{t}$. It is easy to see that the outward normal vector to the ellipsoid lies in the $(s-t)$-plane. If we represent it as $N=c_{s} \partial_{s}+c_{t} \partial_{t}$ then it is clear that the coefficients $c_{s}, c_{t}$ are functions of $r$. Moreover, it is evident from our choice of $\mu$ that $c_{s}\left(r_{0}\right)=0, c_{t}(0)=0$, and that $c_{s}, c_{t}>0$ otherwise.

The second fundamental form $I I(u, v)$ is defined by $I I(u, v)=-g\left(\nabla_{u} v, N\right)$. Thus in order to show positive definiteness it suffices to establish that the components of $\nabla_{u} u$ in the $\partial_{s}$ and $\partial_{t}$ directions are non-positive, at least one of the coefficients is negative for all $r \in\left(0, r_{0}\right)$, at $r=0$ (where $c_{t}=0$ ) we need the coefficient of $\partial_{s}$ to be negative, and at $r=r_{0}$ (where $c_{s}=0$ ) we need the coefficient of $\partial_{t}$ to be negative. (Of course if $u \in T S^{m-1}$ then we must automatically have $r>0$ else this sphere is not defined, and similarly we need $r<r_{0}$ if $u \in T S^{n-1}$.)

Consider first $\partial_{a} \in T S^{m-1}$. From the covariant derivative expressions above we observe that the coefficient of $\partial_{s}$, namely $-\alpha^{\prime} \alpha$, is non-positive and strictly negative for all $r \in\left(0, r_{0}\right)$, however it vanishes at $r=r_{0}$. (We have $r>0$ in order for the vector $\partial_{a}$ to make sense, as noted above.) The coefficient of $\partial_{t}$ is $-\delta^{\prime} \delta \alpha^{2} \gamma^{-2}$, and this is clearly non-negative, but negative at $r=r_{0}$ since $\delta^{\prime}\left(t_{0}\right)>0$ by definition. Thus we have $I I\left(\partial_{a}, \partial_{a}\right)<0$ as required. Analogous arguments apply for $I I\left(\partial_{b}, \partial_{b}\right)$.

Next, we investigate $\nabla_{T} T$. We have

$$
\begin{aligned}
\nabla_{T} T= & \mu_{s}^{\prime}\left(\partial_{s} \mu_{s}^{\prime}\right) \partial_{s}+\mu_{s}^{\prime 2} \nabla_{\partial_{s}} \partial_{s}+\mu_{s}^{\prime}\left(\partial_{s} \mu_{t}^{\prime}\right) \partial_{t}+\mu_{s}^{\prime} \mu_{t}^{\prime} \nabla_{\partial_{s}} \partial_{t} \\
& +\mu_{t}^{\prime}\left(\partial_{t} \mu_{s}^{\prime}\right) \partial_{s}+\mu_{t}^{\prime} \mu_{s}^{\prime} \nabla_{\partial_{t}} \partial_{s}+\mu_{t}^{\prime}\left(\partial_{t} \mu_{t}^{\prime}\right) \partial_{t}+\mu_{t}^{\prime 2} \nabla_{\partial_{t}} \partial_{t}
\end{aligned}
$$

In order to simplify this expression, we note that by definition of $\mu$, the coordinate functions $\mu_{s}(r)$ and $\mu_{t}(r)$ are one-to-one, and therefore invertible. Viewing $s$ as a function of $r$ along $\mu$ we clearly 
have $s(r)=\mu_{s}(r)$, and hence $r(s)=\mu_{s}^{-1}(s)$. Differentiating with respect to $s$ then yields

$$
\begin{aligned}
\partial_{s} \mu_{s}^{\prime}(r) & =\partial_{s} \mu_{s}^{\prime}\left(\mu_{s}^{-1}(s)\right) \\
& =\mu_{s}^{\prime \prime}\left(\mu_{s}^{-1}(s)\right) \frac{1}{\mu_{s}^{\prime}\left(\mu^{-1}(s)\right)} \\
& =\mu_{s}^{\prime \prime}(r) / \mu_{s}^{\prime}(r) .
\end{aligned}
$$

Analogous computations give

$$
\partial_{s} \mu_{t}^{\prime}=\mu_{t}^{\prime \prime} / \mu_{s}^{\prime}, \quad \partial_{t} \mu_{s}^{\prime}=\mu_{s}^{\prime \prime} / \mu_{t}^{\prime}, \quad \partial_{t} \mu_{t}^{\prime}=\mu_{t}^{\prime \prime} / \mu_{t}^{\prime} .
$$

It follows immediately that

$$
\mu_{s}^{\prime}\left(\partial_{s} \mu_{s}^{\prime}\right)=\mu_{s}^{\prime \prime}, \quad \mu_{s}^{\prime}\left(\partial_{s} \mu_{t}^{\prime}\right)=\mu_{t}^{\prime \prime}, \quad \mu_{t}^{\prime}\left(\partial_{t} \mu_{s}^{\prime}\right)=\mu_{s}^{\prime \prime}, \quad \mu_{t}^{\prime}\left(\partial_{t} \mu_{t}^{\prime}\right)=\mu_{t}^{\prime \prime}
$$

Notice that for the above calucations to be valid as stated, we must assume that $\mu_{s}^{\prime}, \mu_{t}^{\prime} \neq 0$. This is fine precisely when $r \in\left(0, r_{0}\right)$. However, observe that the right-hand sides of the expressions (4.4) are defined for all $r \in\left[0, r_{0}\right]$, and we can infer from this that the limits as $r \rightarrow 0^{+}$and $r \rightarrow r_{0}^{-}$in (4.3) must be well-behaved.

We can now use the above calculations to simplify the expression for $\nabla_{T} T$ :

$$
\begin{aligned}
\nabla_{T} T= & \partial_{s}\left(2 \mu_{s}^{\prime \prime}-\mu_{s}^{\prime 2} \frac{\delta^{\prime} \delta}{\gamma^{2}}+2 \mu_{s}^{\prime} \mu_{t}^{\prime} \frac{\delta^{\prime}}{\delta} \partial_{s}\right) \\
& +\partial_{t}\left(2 \mu_{t}^{\prime \prime}-\mu_{t}^{\prime 2} \frac{\gamma^{\prime} \gamma}{\delta^{2}}+2 \mu_{s}^{\prime} \mu_{t}^{\prime} \frac{\gamma^{\prime}}{\gamma}\right)
\end{aligned}
$$

In each of the above brackets, notice that the terms are negative, non-positive and non-positive respectively. It follows that $I I(T, T)>0$ as required.

It remains, then to consider 'mixed' terms, that is $I I(v, w)$ where $v$ and $w$ belong to two of the three basic directions in $\partial E$, namely $T S^{m-1}, T S^{n-1}$ and $\operatorname{Span}\{T\}$. We see from the covariant derivative expressions above that $\nabla_{v} w=\nabla_{w} v=0$ if $v \in T S^{m-1}$ and $w \in T S^{n-1}$, and hence in this case we have $I I(v, w)=0$. For this $v$ and $w$ we also have $\nabla_{\partial_{t}} v=\frac{\delta^{\prime}}{\delta} v$ and $\nabla_{\partial_{t}} w=\frac{\delta^{\prime}}{\delta} w$, and so $I I\left(\partial_{t}, v\right)=-\frac{\delta^{\prime}}{\delta} g(v, N)=0$. Similarly with $\partial_{s}$ in place of $\partial_{t}$. Thus the mixed terms of $I I$ all vanish, and hence we can conclude that $I I$ is positive definite, as required.

Let us summarise the above constructions:

Proposition 1. There is a Ricci positive metric $g$ on $D^{m} \times D^{n}$ and a codimension zero solid ellipsoid $\mathbf{E} \subset D^{m} \times D^{n}$ such that $\partial \mathbf{E}$ is a smooth submanifold of $D^{m} \times D^{n}$ and the normal curvatures of $\partial \mathbf{E}$ (with respect to the outward pointing normal) are all positive.

4.5. Proof of the Main Theorem. Recall from section 1 that to establish the theorem it suffices to construct a fiberwise Ricci positive metric on each Hatcher sphere bundle. In order to do this, we will begin by reconsidering the construction of the Hatcher disc bundle from section 3 .

Now we switch to the relevant notations, i.e., $m=p+1$ and $n=q$. For each point $y \in D_{+}^{4 k}$ we have

$$
D^{p+1} \times D^{q}=P_{y} \cup_{i d} A_{y} \stackrel{\Phi_{y}}{\cong} P_{y} \cup_{\Lambda_{y}} \mathcal{A}_{y}
$$


where we refer the reader to section 3 for the notation. The ellipsoid $\mathbf{E}$ is a subset of $D^{p+1} \times D^{q}$, and so for each $y \in D_{+}^{4 k}$ there is an ellipsoid

$$
\mathbf{E}_{y}:=\Phi_{y}(\mathbf{E}) \subset P_{y} \cup_{\Lambda_{y}} \mathcal{A}_{y} .
$$

Collectively, these ellipsoid fibres form a sub-bundle $\mathcal{E}_{+}^{\text {ell }}$ of $\mathcal{E}_{+}$. Pushing forward the metric $g$ via $\Phi_{y}$ and restricting to $\mathbf{E}_{y}$ equips each $\mathbf{E}_{y}$ with a Ricci positive metric with positive normal curvatures (with respect to the outward normal) at the boundary. Moreover as $y$ varies across $D_{+}^{4 k}$, we obtain in this way a smoothly varying family of fibre metrics on $\mathcal{E}_{+}^{\text {ell }}$.

We similarly form a product bundle $\mathcal{E}_{-}^{\text {ell }} \rightarrow D_{-}^{4 k}$ with total space $D_{-}^{4 k} \times \mathbf{E}$, and take the obvious fiberwise metric where each fibre $\mathbf{E}$ is equipped with the metric induced by $g$. For each fibre $\mathbf{E}_{y} \subset \mathcal{E}_{+}^{\text {ell }}$, notice that we have a decomposition

$$
\mathbf{E}_{y}=\left(\mathbf{E}_{y} \cap P_{y}\right) \cup\left(\mathbf{E}_{y} \cap \mathcal{A}_{y}\right),
$$

and similarly for the fibres of $\mathcal{E}_{-}^{\text {ell }}$.

In order to form the Hatcher disc bundle, we need to glue the bundles $\mathcal{E}_{+}^{\text {ell }}$ and $\mathcal{E}_{-}^{\text {ell }}$ along the boundaries of their base discs. The procedure for gluing the 'full' disc bundles $\mathcal{E}_{+}$and $\mathcal{E}_{-}$is described at the end of section 3. Recall that for each pair of fibres in $\mathcal{E}_{+}$and $\mathcal{E}_{-}$being identified, the annulus parts are identified via the identity map, but the inner 'puck' regions are identified using diffeomorphisms $\tilde{\Lambda}_{y}: P_{y} \rightarrow P_{y}$, which by Lemma 4 split as a product of rotations on the two disc factors. Before proceeding further, we note that these gluing maps restrict to give gluing maps between $\mathcal{E}_{+}^{\text {ell }}$ and $\mathcal{E}_{-}^{\text {ell }}$, since the annulus and puck parts of the respective ellipsoid bundles agree near the boundary of the base discs, and are invariant under rotation of the factors. Note further that by Corollary 2 in section 3 , we do not lose any gluing information by reducing the fibres from the original product of discs considered in section 3 to the ellipsoids currently under consideration. Thus the bundle we will construct using $\mathcal{E}_{+}^{\text {ell }}$ and $\mathcal{E}_{-}^{\text {ell }}$ will be diffeomorphic to that formed from $\mathcal{E}_{+}$ and $\mathcal{E}_{-}$.

From a metric perspective, let us focus first on the puck sub-bundles. As $\Phi_{y}$ is the identity mapping on $P_{y}$, the puck sub-bundle within $\mathcal{E}_{+}^{\text {ell }}$ is just a product, with each fibre equipped with the restriction of $g$. Now the metric $g$ displays rotational symmetry with respect to both disc factors, and so pulling-back $\left.g\right|_{P_{y}}$ via the map $\tilde{\Lambda}_{y}$ results in a metric identical to $\left.g\right|_{P_{y}}$. Since we have set things up so that the metrics near the boundaries of both $\mathcal{E}_{+}$and $\mathcal{E}_{-}$are independent of the radial parameter in the base, we see that gluing the puck sub-bundles along $S^{4 k-1}$ in this way yields a smooth fiberwise metric. (It is perhaps worth remarking that if we were trying to construct a submersion metric on the whole Hatcher disc bundle - as opposed to creating a mere fiberwise metric - then the twisting involved in gluing the bundles $\mathcal{E}_{+}$and $\mathcal{E}_{-}$would have non-trivial metric implications in directions transverse to the fibres.)

Turning our attention to the gluing of the annular regions, we similarly observe that the metric on the annuli close to the boundary of $\mathcal{E}_{+}$is a push-forward via $\Phi_{y}$ of the rotationally symmetric metric $\left.g\right|_{A_{y}}$. Although $\Phi_{y}$ acts non-trivially on the annuli, it nevertheless acts by rotation in both 
$S^{m-1}$ and $S^{n-1}$ directions for $y$ close to $\partial D_{+}^{4 k}$. Thus the pull-back metric on the annuli is identical to the original over the boundary of the base disk, and so gluing the annular part of $\mathcal{E}_{+}$to $\mathcal{E}_{-}$via the identity creates a smooth fiberwise metric in the annular region also.

In summary, we have created a smooth fibrewise Ricci positive metric on the fibres of the Hatcher disc bundle $\mathcal{E}=\mathcal{E}_{+} \cup \mathcal{E}_{-}$. It is immediate that restricting everything in the above argument to the ellipsoid sub-bundles $\mathcal{E}^{\text {ell }}$ - and $\mathcal{E}_{+}^{\text {ell }}$ creates a fiberwise Ricci positive metric on the ellipsoid sub-bundle of the Hatcher disc bundle $\mathcal{E}^{\text {ell }} \subset \mathcal{E}$, with the normal curvatures at the boundary of each fibre being positive with respect to the outer normal.

Finally, we wish to glue two identical copies of the Hatcher disc bundle $\mathcal{E}^{\text {ell }}$ equipped with the above fiberwise metric so as to construct the desired Hatcher sphere bundle. Metrically this is now possible using the family gluing result, Theorem 3, as a consequence of the positive normal curvatures at the boundary. We thus create a Hatcher sphere bundle with a smooth fibrewise Ricci positive metric, as required to establish the theorem.

\section{REFERENCES}

[1] A. Besse, Einstein Manifolds, Springer-Verlag (2002).

[2] B. Botvinnik, J. Ebert, D. J. Wraith, On the topology of the space of Ricci-positive metrics, preprint.

[3] B. Botvinnik, J. Ebert, O. Randal-Williams, Infinite loop spaces and positive scalar curvature, Invent. Math. 209 (2017), no. 3, 749-835.

[4] B. Botvinnik, B. Hanke, T. Schick, M. Walsh, Homotopy groups of the moduli space of metrics of positive scalar curvature, Geom. Topol. 14 (2010), no. 4, 2047-2076.

[5] B. Botvinnik and P.B. Gilkey, The eta invariant and metrics of positive scalar curvature, Math. Ann 302, no. 3, (1995), 507-517.

[6] R. Carr, Construction of manifolds of positive scalar curvature, Trans. A.M.S. 307, no. 1, (1988), 63-74.

[7] D. Crowley, T. Schick, W. Steimle, Harmonic spinors and metrics of positive curvature via the Gromoll filtration and Toda brackets, arXiv:1612.04660v2.

[8] A. Dessai, S. Klaus, W. Tuschmann, Nonconnected moduli spaces of nonnegative sectional curvature metrics on simply-connected manifolds, Bull. London Math. Soc. (to appear).

[9] D. Ebin, The manifold of Riemannian metrics, 1970 Global Analysis (Proc. Sympos. Pure Math., Vol. XV, Berkeley, Calif., 1968) pp. 11-40 Amer. Math. Soc., Providence, R.I.

[10] F. T. Farrell and W. C. Hsiang, On the rational homotopy groups of the diffeomorphism groups of discs, spheres and aspherical manifolds, Algebraic and geometric topology (Proc. Sympos. Pure Math., Stanford Univ., Stanford, Calif., 1976), Part 1, pp. 325-337, Proc. Sympos. Pure Math., XXXII, Amer. Math. Soc., Providence, R.I., 1978.

[11] M. Gromov and H. B. Lawson, Jr., The classification of simply-connected manifolds of positive scalar curvature, Ann. of Math 111 (1980), 423-434.

[12] S. Goette, Morse Theory and Higher Torsion Invariants I, math.DG 0111222

[13] S. Goette, K. Igusa, B. Williams, Exotic smooth structures on topological fiber bundles I, Trans. Amer. Math. Soc. 366 (2014), no. 2, 749-790.

[14] S. Goette, K. Igusa, Exotic smooth structures on topological fiber bundles II, Trans. Amer. Math. Soc. 366 (2014), no. 2, 791-832. 
[15] M.W. Hirsch, Differential Topology, Graduate Texts in Mathematics 33, Springer-Verlag (1976).

[16] B. Hanke, T. Schick and W. Steimle, The Space of Metrics of Positive Scalar Curvature, Publ. Math. Inst. Hautes Études Sci. 120 (2014), 335-367.

[17] M. Kreck, S. Stolz, Nonconnected moduli spaces of positive sectional curvature metrics. J. Amer. Math. Soc. 6 (1993), no. 4, 825-850.

[18] J. Lee Introduction to Smooth Manifolds, Graduate Texts in Mathematics 219, Springer-Verlag (2012).

[19] G. Perelman, Construction of manifolds of positive Ricci curvature with big volume and large Betti numbers, Comparison geometry (Berkeley, CA, 1993-94), 157-163, Math. Sci. Res. Inst. Publ., 30, Cambridge Univ. Press, Cambridge, 1997.

[20] P. Petersen, Riemannian Geometry, Graduate Texts in Mathematics 171, Springer-Verlag (1998).

[21] W. Tuschmann, D. Wraith Moduli Spaces of Riemannian Metrics, Oberwolfach Seminars 46, Birkhauser 2015.

[22] M. Walsh, Metrics of positive scalar curvature and generalised Morse functions, Part II, Trans. Amer. Math. Soc. 366 (2014), no. 1, 1-50.

[23] D.J. Wraith, On the moduli space of positive Ricci curvature metrics on homotopy spheres, Geometry and Topology, 15, 1983-2015.

Department of Mathematics, University of Oregon, Eugene, OR, 97405, USA

E-mail address: botvinn@uoregon.edu

Department of Mathematics and Statistics, National University of Ireland Maynooth, Maynooth, IRELAND

E-mail address: mark.walsh@mu.ie

Department of Mathematics and Statistics, National University of Ireland Maynooth, Maynooth, IRELAND

E-mail address: david.wraith@mu.ie 